\title{
ALGEBRAIC SHIFT EQUIVALENCE AND PRIMITIVE MATRICES
}

\author{
MIKE BOYLE AND DAVID HANDELMAN
}

\begin{abstract}
Motivated by symbolic dynamics, we study the problem, given a unital subring $S$ of the reals, when is a matrix $A$ algebraically shift equivalent over $S$ to a primitive matrix? We conjecture that simple necessary conditions on the nonzero spectrum of $A$ are sufficient, and establish the conjecture in many cases. If $S$ is the integers, we give some lower bounds on sizes of realizing primitive matrices. For Dedekind domains, we prove that algebraic shift equivalence implies algebraic strong shift equivalence.
\end{abstract}

\section{INTRODUCTION}

Unless specified otherwise, we use $S$ to denote an integral domain; it most commonly will be a unital subring of the reals and frequently it will be equipped with the relative ordering, $S_{+}=S \cap[0, \infty)$. A matrix over $S$ is nonnegative if all of its entries lie in $S_{+}$; a matrix is primitive if it is square, nonnegative, and some power is strictly positive. In this paper we consider the following problem: When is a matrix with entries from $S$ algebraically shift equivalent to a primitive matrix over $S$ ? This is one piece of the fundamental problem of understanding the constraints which ordering places on algebraic structure. Our results in this direction, which we summarize at the end of this section, are particularly relevant to symbolic dynamics and linear algebra. Our methods derive from matrix theory, abstract algebra, and symbolic dynamics. We also give some new results of independent interest for algebraic shift equivalence and matrix forms.

The problem involves different areas of mathematics and is new, so we will take some time now to explain it and give some background.

Suppose $\mathscr{A}$ and $\mathscr{B}$ are endomorphisms in some category. Then $\mathscr{A}$ and $\mathscr{B}$ are shift equivalent (in that category) if $\mathscr{A}=\mathscr{B}$ or there exist a positive integer $l$ (the lag) and morphisms $X$ and $Y$ such that the following equations hold:

$$
X Y=\mathscr{A}^{l}, \quad Y X=\mathscr{B}^{l}, \quad X \mathscr{B}=\mathscr{A} X, \quad Y \mathscr{A}=\mathscr{B} Y .
$$

When the lag is one, the equations $\mathscr{A}=X Y, \mathscr{B}=Y X$ imply the others. Shift equivalence is an equivalence relation; in general, lag one shift equivalence is not. The transitive closure of lag one shift equivalence is called strong

Received by the editors November 28, 1990.

1991 Mathematics Subject Classification. Primary 15A48; Secondary 06F25, 58F03, 15A36, $15 \mathrm{A33}$.

The first author was partially supported by NSF Grant DMS-860169.

The second author was supported in part by an operating grant from NSERC (Canada). 
shift equivalence (within the category). Strong shift equivalence implies shift equivalence. When the morphisms in the category are matrices over $S_{+}$or $S$, we use italic letters to denote them. In the former case, we say $A$ and $B$ are shift equivalent or strongly shift equivalent (over $S_{+}$), respectively. When the morphisms are matrices over $S$, we say that $A$ and $B$ are algebraically shift equivalent or algebraically strongly shift equivalent (over $S$ ).

Let $S^{n}$ denote the set of columns of size $n$ with entries from $S$; of course, $S^{n}$ is a free $S$-module of rank $n$. These modules are the objects of a category whose morphisms are $S$-module homomorphisms, which we consider as rectangular matrices over $S$. In particular, suppose $A$ is an $n \times n$ matrix over $S$. Define the direct limit $S$-module $\mathscr{G}(A)$ as the direct limit of the maps

$$
S^{n} \stackrel{A}{\longrightarrow} S^{n} \stackrel{A}{\longrightarrow} S^{n} \stackrel{A}{\longrightarrow} S^{n} \stackrel{A}{\longrightarrow} \cdots .
$$

The elements of $\mathscr{G}(A)$ are the ordered pairs $(b, k)$, where $b$ is an element of $S^{n}$ and $k$ is a positive integer (describing the level, or copy of $S^{n}$, in which $b$ appears), modulo the equivalence relation $[b, k]=\left[b^{\prime}, k^{\prime}\right]$ if there exists $k^{\prime \prime} \geq k, k^{\prime}$ such that $A^{k^{\prime \prime}-k} b=A^{k^{\prime \prime}-k^{\prime}} b^{\prime}$. In particular, $[A b, k+1]=[b, k]$. We observe that $[b, k]$ is zero if and only if $A^{m} b$ is the zero column for some integer $m$; since $A$ is $n \times n$ (and $S$ is a domain), this is equivalent to $A^{n} b=\mathbf{0}$.

The matrix $A$ induces an $S$-module automorphism of $\mathscr{G}(A)$ by means of $\hat{A}$ : $[b, k] \mapsto[A b, k]$; the inverse map is the shift $[b, k] \mapsto[b, k+1]$. We obtain the pair $(\mathscr{G}(A), \widehat{A})$ from $A$, and it is clear from the equations in $(0.1)$ that this pair is an algebraic shift invariant; this means that if $A$ is algebraically shift equivalent to $B$, then there is an $S$-module isomorphism $\phi: \mathscr{G}(A) \rightarrow \mathscr{G}(B)$ such that $\widehat{B} \phi=\phi \widehat{A}$ (in fact, $[b, k]_{A} \mapsto[Y b, k]_{B}$ will do). Conversely, if such an isomorphism $\phi$ exists, it is not difficult to deduce that $A$ and $B$ must be algebraically shift equivalent (or shift equivalent).

There is a particularly concrete presentation of the pair $(\mathscr{G}(A), \widehat{A})$. Let $F$ be the field of fractions of $S$. If $A$ is an $n \times n$ matrix over $S$, regard $A$ as a linear transformation of $F^{n}$. Let $V$ be the image of $F^{n}$ under $A^{n}$. Let $G$ be the set of elements in $V$ mapped by some positive power of $A$ into $S^{n}$. Then the restriction of $A$ to $G$ is a presentation of $(\mathscr{G}(A), \widehat{A})$.

In particular, when $S$ is a field, the algebraic shift equivalence class of a matrix $A$ over $S$ is just the isomorphism class of the linear transformation which is the restriction of $A$ to the maximal subspace on which it is nonsingulargiven, for example, by the nonsingular part of the Jordan form of $A$ over $\mathbf{C}$. For $S=\mathbf{R}$, our problem is closely related to an intractable problem in linear algebra: When is a matrix similar to a nonnegative matrix? For proper subrings of $\mathbf{R}$, there are additional complications.

The relations of algebraic shift equivalence and strong shift equivalence were introduced by Williams [W1] in his classification of one-dimensional attractors in dynamical systems. He invented these relations to provide a tractable finite presentation for automorphisms of inverse limits of tori and other groups; the direct limit modules above are simply the dual objects to his inverse limit systems.

Shifts of finite type (SFT) (also called subshifts of finite type, or topological Markov chains) are important topological dynamical systems which can be defined by nonnegative integral matrices. In the enormously influential paper 
[W2], Williams approached the problem of classifying SFT's up to topological conjugacy by analyzing the shift equivalence and strong shift equivalence of their defining matrices (over $\mathbf{Z}_{+}$). He proved that strong shift equivalence is a complete invariant of topological conjugacy, and conjectured that shift equivalent nonnegative integral matrices must be strongly shift equivalent. (This conjecture was refuted, after some 16 years, in the dramatic recent paper of Kim and Roush [KR3]. However, it remains open in the most important case, where the shift equivalent matrices are primitive; for some partial results see [B2], and also several papers of Kim and Roush.)

In any case, understanding shift equivalence remains an essential step in the classification of SFT's, and it turns out that aspects of shift equivalence are crucial for analyzing automorphisms and homomorphisms of SFT's (see, for example, [As, BMT, KMT, KR3]). The algebraic analysis of shift equivalence naturally begins with not necessarily nonnegative matrices; for primitive matrices (the main case), Parry and Williams [PW] (also see [KR1, Theorem 2.1]) showed that algebraic shift equivalence implies shift equivalence, so there is no further step. For a classification theory, one wants to know "which" matrices occur; this is also important for constructions and counterexamples in the theory of coding relations of SFT's. Thus we are led to the problem: Determine which algebraic shift equivalence classes of integral matrices are realized by (i.e., contain) a primitive matrix. This is the root of our problem in symbolic dynamics.

We now recapitulate the two conjectures of $[\mathrm{BH}]$, which we hope describe the solution to our problem. A list of complex numbers (including repetitions) will be denoted $\Delta=\left(d_{1}, d_{2}, \ldots, d_{k}\right)$. When we say " $\Delta$ is the nonzero spectrum of a matrix $A$ ", we mean the nonzero part of $\Delta$ is the nonzero part of the spectrum of $A$, counting multiplicities. We define $\operatorname{tr} \Delta^{n}=\sum d_{i}^{n}$, that is, the trace of the $n$th power of the diagonal matrix whose diagonal entries are the entries of $\Delta$. Let $\mu$ denote the Möbius function; i.e., $\mu: \mathbf{N} \rightarrow\{0, \pm 1\}$ is given by

$$
\mu(n)= \begin{cases}0 & \text { if } n \text { is divisible by a nontrivial square, } \\ (-1)^{s} & \text { if } n \text { is square-free with exactly } s \text { prime divisors. }\end{cases}
$$

We define $\operatorname{tr}_{n}(\Delta)=\sum_{k \mid n} \mu\left(\frac{n}{k}\right) \operatorname{tr}\left(\Delta^{k}\right)$ to be the nth net trace of $\Delta$.

Spectral Conjecture. Let $S$ be a unital subring of $\mathbf{R}$. The list $\Delta$ is the nonzero spectrum of a primitive matrix over $S$ if and only if

(1) there exist $d=d_{j}$ in $\Delta$ such that $d=|d|>\left|d_{i}\right|$ for all $i \neq j$;

(2) the coefficients of the polynomial (in the variable $t) \prod\left(t-d_{i}\right)$ all belong to $S$

(3) if $S=\mathbf{Z}$, then $\operatorname{tr}_{n}(\Delta) \geq 0$ for all positive integers $n$;

$\left(3^{\prime}\right)$ if $S \neq \mathbf{Z}$, for all positive integers $n$ and $k, \operatorname{tr} \Delta^{n} \geq 0$, and if $\operatorname{tr} \Delta^{n}>0$, then $\operatorname{tr} \Delta^{n k}>0$.

Condition (2) is expressible as, "all symmetric functions on $\Delta$ take values in $S$ ". As observed in [BH], the necessity of these conditions is clear. Sufficiency was proved if the largest number $d$ is either in $S$ or satisfies a quadratic equation over $S$. (A more general result was established.) In particular, this applies if $S$ is one of the reals, the algebraic real numbers, or the algebraic 
integers, among others. As a consequence of work with Kim and Roush [BH, Appendix 4], if $S \neq \mathbf{Z}$, the conditions of the spectral theorem are satisfied, and if $\operatorname{tr} \Delta>0$, then there exists a strictly positive matrix over $S$ realizing $\Delta$ as its nonzero spectrum.

Generalized Spectral Conjecture. Let $A$ be a square matrix with entries from $S$. Then $A$ is algebraically shift equivalent over $S$ to a primitive matrix if and only if the nonzero spectrum satisfies the conditions of the Spectral Conjecture.

We now turn to the statement of our results. In $\S 1$, we give the basic results on algebraic shift equivalence and matrix forms which we need for the realization results of $\S 3$. In $\S 2$, we consider matrices over Dedekind domains, and show for these that algebraic shift equivalence implies algebraic strong shift equivalence. The proof involves projective modules, and we ask if it can be generalized to matrices over rings with finite projective dimension (such as matrices over Laurent polynomials with integral coefficients; their properties related to shift equivalence are crucial for the analysis of codes between Markov chains [MT]). We give examples of matrices over Dedekind domains which are not algebraically shift equivalent to nonsingular matrices-this contrasts with the situation for principal ideal domains. Finally we prove an analogue of a classical result on principal ideal domains for Dedekind domains. Specifically, if $p_{1} \cdots \cdots p_{n}$ is a factorization of the characteristic polynomial $\chi_{A}(t)$ for the matrix $A$ over $S$ in the polynomial ring $S[t]$, then $A$ is GL $(\cdot, S)$-conjugate to a matrix $B$ in block triangular form with diagonal blocks $B_{1}, \ldots, B_{n}$ such that $\chi_{B_{i}}(t)=p_{i}(t)$. For $S$ a Dedekind domain, this can fail; however, we show that $A$ is algebraically shift equivalent over $S$ to some block triangular matrix with diagonal blocks $B_{1}, \ldots, B_{n}$ for which $\chi_{B_{i}}(t)$ equals either $p_{i}(t)$ or $t p_{i}(t)$. We use this triangular form in $\S 3$, where we prove our central (technical) theorem (Theorem 3.1), and use it to verify the Generalized Spectral Conjecture in several cases:

- when the nonzero spectrum is contained in $S$, and either $S=\mathbf{Z}$ or $S$ is a Dedekind domain with a nontrivial unit (3.3);

- when $S$ is dense, $A$ has positive trace, and either

$\diamond$ the spectrum is real, or

$\diamond$ the minimal and characteristic polynomials of $A$ are equal up to a power of the indeterminate (e.g., the characteristic polynomial of $A$ has no repeated nonzero roots).

One can think of the last result as a verification of the generalized spectral conjecture for dense subrings $S$ of $\mathbf{R}$ in the generic case. We offer a conjecture (the Radius Conjecture) which in particular would let us apply Theorem 3.1 much more generally. We also pose the natural realization question for symmetric matrices.

In $\S 4$, we consider integral matrices whose nonzero spectrum consists of two integers satisfying the necessary conditions of the Spectral Conjecture, and show that any such matrix is algebraically shift equivalent over $\mathbf{Z}$ to a primitive matrix of size 4 or less. In $\S 5$, we consider some lower bounds to the minimum size of a primitive integral matrix representing a given algebraic shift equivalence class. 


\section{Algebraic Shift equivalence}

Suppose that $A$ is an $n \times n$ matrix over a unital ring $S$. Let $\mathscr{E}(A)$ denote the endomorphism ring of $(\mathscr{G}(A), \widehat{A})$ : This is the ring of $S$-module endomorphisms of $\mathscr{G}(A)$ that commute with $\hat{A}$; equivalently, it is the ring of endomorphisms of $\mathscr{G}(A)$ as a module over $S[\widehat{A}]$. Define $C_{S}(A)$ as the centralizer of $A$ in $M_{n} S$,

$$
C_{S}(A)=\left\{C \in M_{n} S \mid A C=C A\right\} .
$$

An element $C$ of $C_{S}(A)$ induces an element of $\mathscr{E}(A)$ by means of the rule $[b, k] \mapsto[C b, k]$. This yields a homomorphism of $S$-rings, $C_{S}(A) \rightarrow \mathscr{E}(A)$. Abusing notation slightly, we let $U(A)$ denote the set of elements of $C_{S}(A)$ that map to units of $\mathscr{E}(A)$. A matrix $C$ in $C_{S}(A)$ belongs to $U(A)$ if and only if there exist an integer $k$ and a matrix $D$ in $C_{S}(A)$ (this can be weakened to $D$ in $M_{n} S$ ) such that $C D=A^{k}$. It is possible to show that $\mathscr{E}(A)$ is the ring obtained from the image of $C_{S}(A)$ by inverting $\hat{A}$.

Given square matrices $A$ and $B$ of sizes $m$ and $n$ respectively, define

$$
[A, B]_{S}=\left\{A T-T B \mid T \in S^{m \times n}\right\} .
$$

Proposition 1.1 (cf. [H2, III.4]). Let $A$ and $B$ be square matrices over a unital ring $S$, and let $X$ and $X^{\prime}$ be rectangular matrices of the appropriate dimensions, with entries from $S$. Suppose there exist matrices $\{E, F\} \subset U(A)$ and $\{G, H\} \subset U(B)$, such that $E X G-F X^{\prime} H \in[A, B]_{S}$. Then the block upper triangular matrices

$$
C=\left[\begin{array}{cc}
A & X \\
\mathbf{0} & B
\end{array}\right], \quad C^{\prime}=\left[\begin{array}{cc}
A & X^{\prime} \\
\mathbf{0} & B
\end{array}\right]
$$

are algebraically shift equivalent over $S$. If $X-X^{\prime} \in[A, B]_{S}$ then $C$ and $C^{\prime}$ are $\mathrm{SL}(\cdot, S)$-conjugate.

Proof. First, if $X-X^{\prime}=A T-T B$ for $T$ a rectangular matrix with entries from $S$, define $Y=\left[\begin{array}{ll}I & T \\ 0 & I\end{array}\right]$, and compute $C=Y C^{\prime} Y^{-1}$. In particular, $C$ is algebraically shift equivalent to $C^{\prime}$.

Next, suppose that $E E_{0}=A^{t}$ for some integer $t$; by replacing $E_{0}$ by $A^{n} E_{0}$, we can assume that $E_{0}$ commutes with $A$. Let $U$ be the upper right block of $C^{t}$; then $U=\sum_{i=0}^{t-1} A^{i} X B^{t-i-1}$. Now set $R=\left[\begin{array}{cc}E & 0 \\ 0 & I\end{array}\right], W=\left[\begin{array}{cc}E_{0} & U \\ 0 & B^{t}\end{array}\right]$. Then

$$
\begin{aligned}
& R W=\left[\begin{array}{cc}
A^{t} & E U \\
\mathbf{0} & B^{t}
\end{array}\right]=\left[\begin{array}{cc}
A & E X \\
\mathbf{0} & B
\end{array}\right]^{t}, \\
& W R=\left[\begin{array}{cc}
A^{t} & U \\
\mathbf{0} & B^{t}
\end{array}\right]=\left[\begin{array}{cc}
A & X \\
\mathbf{0} & B
\end{array}\right]^{t} .
\end{aligned}
$$

A similar computation reveals that if $Z=\left[\begin{array}{cc}A & E X \\ 0 & B\end{array}\right]$, then $R C=Z R$ and $W Z=$ $C W$. Hence $C$ is algebraically shift equivalent to $Z$. Applying the same process on the other side, we see that if $Z^{\prime}=\left[\begin{array}{ccc}A & E X G \\ 0 & B\end{array}\right]$, then $Z$ and $Z^{\prime}$ are algebraically shift equivalent. By the result in the first paragraph of this proof, $Z^{\prime}$ is algebraically shift equivalent to $\left[\begin{array}{cc}A F X^{\prime} H \\ 0 & B\end{array}\right]$, which is in turn algebraically shift equivalent to $C^{\prime}$.

A useful partial converse exists. 
Proposition 1.2. Let $A$ and $B$ be square matrices over $S$ such that the intersection of their spectra is either empty or consists of 0 (with arbitrary multiplicity). Suppose that $X$ and $X^{\prime}$ are rectangular matrices over $S$ such that

$$
C=\left[\begin{array}{cc}
A & X \\
\mathbf{0} & B
\end{array}\right], \quad C^{\prime}=\left[\begin{array}{cc}
A & X^{\prime} \\
\mathbf{0} & B
\end{array}\right]
$$

are algebraically shift equivalent over $S$. Then there exist matrices $R$ in $U(A)$ and $P$ in $U(B)$ such that $R X^{\prime}-X P$ belongs to $[A, B]_{S}$.

Proof. Let $V=\left[\begin{array}{ll}R & W \\ T & P\end{array}\right]$ be an intertwining matrix for $C$ and $C^{\prime}$. Calculation of the block $(2,1)$ entry of the products reveals that $B T=T A$. The hypothesis on the spectra forces, via an elementary argument, $T A^{n}=\mathbf{0}$ for some $n$. Since we may replace $V$ by $V\left(C^{\prime}\right)^{n}$, we may assume $T=\mathbf{0}$. Now let $V^{\prime}=\left[\begin{array}{cc}R^{\prime} & W^{\prime} \\ T^{\prime} & P^{\prime}\end{array}\right]$ satisfy $V C^{\prime}=C^{\prime} V^{\prime}, V^{\prime} V=\left(C^{\prime}\right)^{k}$ and $V V^{\prime}=C^{k}$. We similarly may assume that $T^{\prime}=0, R R^{\prime}=A^{k}=R^{\prime} R$, and $P P^{\prime}=B^{k}=P^{\prime} P$. From $C V=V C^{\prime}$, it follows that $R A=A R$ and $P B=B P$. Hence the images of $R$ and $P$ are units in the corresponding endomorphism rings. Computing the $(1,2)$ block entries of $C V=V C^{\prime}$, we obtain $R X^{\prime}-X P=A W^{\prime}-W^{\prime} B$, finishing the proof.

The hypothesis that the spectra be disjoint is equivalent to the operator $T_{A, B}: S^{m \times n} \rightarrow S^{m \times n}(z \mapsto A z-z B)$ being one-to-one. Its cokernel is thus a torsion module over $S$. If $S$ is a field, this forces the cokernel to be zero, and thus there is only one algebraic shift equivalence class. If $S=\mathbf{Z}$, the cokernel is finite, so there are at most finitely many classes.

In general, given $A$ and $B$, the space of algebraic shift equivalence classes over $S$ of $\left[\begin{array}{ll}A & X \\ 0 & B\end{array}\right]$ is the orbit space of the action of $U(A) \times U(B)$ on the quotient module, the cokernel of $T_{A, B}$.

Blocking Lemma 1.3. Suppose that $\sim$ represents one of the following relations on square matrices over the ring $S:$ lag $l$ shift equivalence; strong shift equivalence; lag $l$ algebraic shift equivalence; algebraic strong shift equivalence; or conjugacy via $\mathrm{GL}(\cdot, S)$. Let $A_{i}$ and $A_{i}^{\prime}(i=1,2, \ldots, n)$ be matrices over $S$, such that for all $i, A_{i} \sim A_{i}^{\prime}$. Suppose $A$ is a block upper triangular matrix whose diagonal blocks are $A_{1}$ through $A_{n}$. Then there exists a block upper triangular matrix $A^{\prime}$ over $S$ with diagonal blocks $A_{1}^{\prime}$ through $A_{n}^{\prime}$ such that $A \sim A^{\prime}$.

Proof. For conjugacy, given $U_{i} A_{i} U_{i}^{-1}=A_{i}^{\prime}$, let $U=\operatorname{diag}\left(U_{1}, \ldots, U_{n}\right)$ and $A^{\prime}=U A U^{-1}$. Among the other relations, we give the argument for shift equivalence; the argument for algebraic strong shift equivalence is the same and the strong relations follow from the lag 1 case.

By induction, it suffices to prove the result in the case that $n=2$. It is convenient to change notation, so $B, C$, and $D$ are square matrices and $X$ is rectangular. Suppose that $B^{l}=T R, C^{l}=R T, B T=T C$, and $R B=C R$. Define $Y$ so that

$$
\left[\begin{array}{ll}
B & X \\
\mathbf{0} & D
\end{array}\right]^{l}=\left[\begin{array}{cc}
B^{l} & Y \\
\mathbf{0} & D^{l}
\end{array}\right]
$$

Then $\left[\begin{array}{ll}B & X \\ 0 & D\end{array}\right]$ is lag $l$ algebraically shift equivalent to $\left[\begin{array}{ll}C & R X \\ 0 & D\end{array}\right]$ via $\left[\begin{array}{ll}R & 0 \\ 0 & 1\end{array}\right]$ and $\left[\begin{array}{ll}T & Y \\ 0 & D^{l}\end{array}\right]$. Similarly, $\left[\begin{array}{ll}D & X \\ 0 & B\end{array}\right]$ is lag $l$ algebraically shift equivalent to $\left[\begin{array}{lll}D & X T \\ 0 & C\end{array}\right]$ via 
$\left[\begin{array}{ll}I & 0 \\ 0 & T\end{array}\right]$ and $\left[\begin{array}{ll}D^{l} & Z \\ 0 & R\end{array}\right]$, where $Z$ is the $(1,2)$ block entry of $\left[\begin{array}{ll}D & X \\ 0 & B\end{array}\right]^{l}$.

Theorem 1.4 [N, Chapter III, §15]. Let $A$ be a square matrix over a principal ideal domain $S$. Let $p_{1} p_{2} \cdots p_{m}$ be a factorization of the characteristic polynomial of $A$, where each $p_{i}$ is monic and with coefficients from $S$. Then $A$ is conjugate via $\mathrm{GL}(\cdot, S)$ to a matrix $C$ in block upper triangular form, wherein the characteristic polynomial of the ith block is $p_{i}$.

Proposition 1.5. Suppose that $C=\left[\begin{array}{ll}B & X \\ 0 & N\end{array}\right]$ is a block triangular matrix over $S$ and $N$ is nilpotent and upper triangular. Then $C$ is algebraically strongly shift equivalent over $S$ to $B$.

Proof. The bottom row of $C$ is zero. We may thus rewrite

$$
C=\left[\begin{array}{cc}
B^{\prime} & X^{\prime} \\
\mathbf{0} & 0
\end{array}\right]
$$

and use the elementary algebraic strong shift equivalence,

$$
\left[\begin{array}{cc}
B^{\prime} & X^{\prime} \\
\mathbf{0} & 0
\end{array}\right]=\left[\begin{array}{l}
\mathbf{I} \\
0
\end{array}\right]\left[\begin{array}{ll}
B^{\prime} & X^{\prime}
\end{array}\right], \quad B^{\prime}=\left[\begin{array}{ll}
B^{\prime} & X^{\prime}
\end{array}\right]\left[\begin{array}{l}
\mathbf{I} \\
0
\end{array}\right] .
$$

This reduces the size, and we continue until the resulting matrix is nonsingular.

Corollary 1.6 [Ef]. A square matrix over a principal ideal domain is algebraically strongly shift equivalent to a nonsingular matrix.

Proof. Apply Theorem 1.4 and Proposition 1.5.

(Effros showed that Corollary 1.6 holds for matrices over the integers, but his proof works for all principal ideal domains.) The following simple application of the results above is quite useful for constructing examples in symbolic dynamics (see, for example, [Fi, Example 3.11; BLR, Example 4.2].

Proposition 1.7. Suppose that $n$ and $k$ are nonzero integers. Then every integral matrix with nonzero spectrum $(n, k)$ is algebraically strongly shift equivalent over $\mathbf{Z}$ to a matrix of the form $C_{m}=\left[\begin{array}{ll}n & m \\ 0 & k\end{array}\right]$. The algebraic shift equivalence class of $C_{m}$ is determined by the equivalence relation on $\{m \in \mathbf{Z}\}$ generated by

$$
\begin{array}{ll}
m \sim m^{\prime} & \text { if } m \equiv m^{\prime} \bmod n-k, \\
m \sim p m & \text { if } p \mid n \text { or } p \mid k .
\end{array}
$$

Proof. The form for $C_{m}$ comes from Theorem 1.4. For $n \neq k$, the rest comes from Proposition 1.1 and Proposition 1.2. A similar argument works if $n=k$.

The following generalizes [H2, III.4]:

Lemma 1.8. Let $A$ and $B$ be square, real matrices. Suppose there exists an eigenvalue, $\rho$, of $A$ that is not an eigenvalue of $B$, such that $A$ admits a strictly positive right eigenvector for $\rho$. Then there exists a rectangular matrix $X$ with integer entries such that $A X-X B$ is strictly positive. If $B$ is a nonnegative matrix and $\rho$ exceeds the spectral radius of $B$, then we may find $X$ to additionally be strictly positive.

Proof. Let $w$ denote the strictly positive right eigenvector for $A$ corresponding to $\rho$. Suppose that $A$ is of size $n$, and $B$ is of size $m$. Let $Z$ be any strictly 
positive $1 \times m$ matrix. Define $X_{0}=w Z(\rho \mathbf{I}-B)^{-1}$. Then we have

$$
\begin{aligned}
A X_{0}-X_{0} B & =\rho w Z(\rho \mathbf{I}-B)^{-1}-w Z(\rho \mathbf{I}-B)^{-1} B \\
& =w Z(\rho \mathbf{I}-B)^{-1}(\rho \mathbf{I})-w Z(\rho \mathbf{I}-B)^{-1}(B) \\
& =w Z(\rho \mathbf{I}-B)^{-1}(\rho \mathbf{I}-B)=w Z \gg \mathbf{0} .
\end{aligned}
$$

If $X_{1}$ in $\mathbf{Q}^{n \times m}$ is sufficiently close to $X_{0}$, then $A X_{1}-X_{1} B \gg \mathbf{0}$. There exists a positive integer $N$ so that $X=N X_{1}$ has only integer entries. If $B$ is nonnegative and its spectral radius is dominated by $\rho$, then $(\rho \mathbf{I}-B)^{-1}$ is nonnegative, so we may choose the original $X_{0}$ to be strictly positive itself.

Corollary 1.9. Let $S$ be a unital subring of $\mathbf{R}$. Suppose that $A$ and $B$ are square matrices of sizes $n$ and $m$ respectively, with entries from $S$. If $A$ is primitive and its spectral radius is not an eigenvalue of $B$, there exists a strictly positive $n \times m$ matrix $H$ (with entries from $S$ ) such that for all $n \times m$ matrices $J$ over $S$, the matrices

$$
\left[\begin{array}{ll}
A & J \\
\mathbf{0} & B
\end{array}\right] \quad \text { and } \quad\left[\begin{array}{cc}
A & J+H \\
\mathbf{0} & B
\end{array}\right]
$$

are similar (and thus algebraically shift equivalent) over $S$.

Proof. Let $X$ be as in the conclusion of Lemma 1.8, and set $H=A X-X B$; since all of the entries of $X$ lie in $\mathrm{Z}$, those of $H$ lie in $S$. The result now follows from Proposition 1.1.

By a nilpotent Jordan block, we mean a square zero-one matrix $J$ with $J(i, j)=1$ if and only if $j=i+1$. In particular, if $J$ has size one, then $J=[0]$. By a nilpotent Jordan matrix, we mean a direct sum of nilpotent Jordan blocks. The following can also be deduced from arguments in the proof of [KR4, 3.2].

Theorem 1.10. Suppose that $S$ is a unital nondiscrete subring of $\mathbf{R}$, and $A$ is a strictly positive square matrix with entries from $S$. Let $N$ be the nilpotent part of the (real) Jordan form for $A$. Suppose that $N^{\prime}$ is a nilpotent Jordan matrix for which there is a one-to-one map from the set of nilpotent Jordan blocks (with multiplicities) of $N$ to that of $N^{\prime}$ that does not decrease the size of any block. Then $A$ is strongly shift equivalent (over $S_{+}$) to a strictly positive matrix $A^{\prime}$ which admits $N^{\prime}$ as the nilpotent part of its Jordan form.

Proof. By induction, it suffices to consider the case $N^{\prime}=\left[\begin{array}{ll}N & x \\ 0 & 0\end{array}\right]$, where $x$ is a zero-one column vector. There is a nonsingular matrix $U$ with entries from the field of fractions of $S$ such that $U^{-1} A U$ has the block form $\left[\begin{array}{ll}B & C \\ 0 & N\end{array}\right]$. We may assume that $U$ (but not $U^{-1}$ of course) has all of its entries in $S$. For any element $\varepsilon$ in $S \cap \mathbf{R}_{+}$, the matrix

$$
A_{1}=\left[\begin{array}{ccc}
B & C & \mathbf{0} \\
\mathbf{0} & N & \varepsilon x \\
\mathbf{0} & \mathbf{0} & 0
\end{array}\right]
$$

is a matrix over $S$ with the required Jordan form. Let $y$ be the column vector $U\left[\begin{array}{l}0 \\ x\end{array}\right]$, so

$$
\left[\begin{array}{ll}
U & \mathbf{0} \\
\mathbf{0} & 1
\end{array}\right] A_{1}\left[\begin{array}{cc}
U^{-1} & \mathbf{0} \\
\mathbf{0} & 1
\end{array}\right]=\left[\begin{array}{cc}
A & \varepsilon y \\
\mathbf{0} & 0
\end{array}\right] .
$$


Let $v$ be the column vector all of whose entries are 1 , and set $w=v^{\mathrm{T}}$. Now $z=A v$ is a strictly positive column. For $\delta$ an element of $S$ such that $0<$ $\varepsilon<\delta$ and $\delta / \varepsilon$ is sufficiently large, the column $\varepsilon y+\delta z$ is strictly positive. The matrix $A_{2}=\left[\begin{array}{cc}A & \varepsilon y+\delta z \\ 0 & 0\end{array}\right]$ is strongly shift equivalent (over $S_{+}$) to $A$ (Proposition 1.5). We may choose $\varepsilon$ and $\delta$ so small (but keeping their ratio fixed) so that $A-(\varepsilon y+\delta z) w$ is also strictly positive. Define

$$
R=\left[\begin{array}{cc}
A & \varepsilon y+\delta z \\
\mathbf{0} & 0
\end{array}\right]\left[\begin{array}{cc}
\mathbf{I} & \mathbf{0} \\
-w & 1
\end{array}\right], \quad T=\left[\begin{array}{cc}
\mathbf{I} & \mathbf{0} \\
w & 1
\end{array}\right] .
$$

Then $R$ and $T$ yield a lag 1 strong shift equivalence and a similarity between $A_{2}$ and a strictly positive matrix $A^{\prime}$. However, $A_{2}$ is similar to $\left[\begin{array}{cc}A & \varepsilon y \\ 0 & 0\end{array}\right]$ via $\left[\begin{array}{ll}\mathbf{I} & v \\ \mathbf{0} & 1\end{array}\right]$.

\section{MATRICES OVER DEDEKIND DOMAINS}

Effros showed that a square matrix over a principal ideal domain $S$ is algebraically strongly shift equivalent over $S$ to a nonsingular matrix [Ef] (see Corollary 1.6). We begin with an easy example to show that even when the coefficient ring is a Dedekind domain, a matrix need not be algebraically shift equivalent to a nonsingular matrix. The obstruction is just the existence of projective modules that are not free.

Proposition 2.1. Let $S$ be a commutative domain admitting a finitely generated projective module $P$ that is not free. From a module $Q$ such that $P \oplus Q \simeq S^{n}$, let $e: S^{n} \rightarrow S^{n}$ be the endomorphism that projects onto $P$. Then any matrix representation for $e$ is not algebraically shift equivalent over $S$ to a nonsingular matrix.

Proof. Such a $Q$ exists since $P$ is finitely generated and projective. We observe that $e^{2}=e$, so all of the nonzero eigenvalues of the latter (as an endomorphism of the free $S$-module) are 1 . Hence if it were algebraically shift equivalent to a nonsingular matrix, that matrix would have to be the identity of size equalling the rank of $P$. From the definitions, it follows that an algebraic shift equivalence between these matrices would entail an $S$-module isomorphism on their corresponding ranges. This would force $P$ to be free, a contradiction.

Every Dedekind domain that is not a principal ideal domain of course admits a nonfree projective; we give an explicit example of the corresponding matrix.

Example 2.2. A matrix not algebraically shift equivalent to a nonsingular matrix.

Let $S=\mathbf{Z}[\sqrt{15}]$ and let $A$ and $A^{\prime}$ be the matrices

$$
\left[\begin{array}{cc}
-5 & 2 \sqrt{15} \\
-\sqrt{15} & 6
\end{array}\right], \quad\left[\begin{array}{cc}
7 \sqrt{15}-27 & 66-15 \sqrt{15} \\
3 \sqrt{15}-11 & 28-7 \sqrt{15}
\end{array}\right]
$$

We observe that $A^{2}=A$ and its eigenvalues are 0 and 1 . We take the nonprincipal ideal $I=(3, \sqrt{15})$ of $S$, and set $J=(5, \sqrt{15})$. Then we verify that the direct sum $I \oplus J$ is a free $S$-module with $S$-basis the set of column vectors

$$
\left\{(\sqrt{15},-\sqrt{15})^{\mathrm{T}},(-6,5)^{\mathrm{T}}\right\}
$$


and the projection onto $I$ has matrix $A$ in terms of this basis. As the class group of $\mathbf{Z}[\sqrt{15}]$ is of order 2 , the matrix $A \oplus A$ is algebraically shift equivalent to the $2 \times 2$ identity matrix. The matrix $A^{\prime}$ is strictly positive (as a real matrix) and is conjugate to $A$.

Williams [W1, Lemma 4.6] gave an argument which showed in many categories that shift equivalence implies strong shift equivalence. Viewed as a proof that algebraic shift equivalence implies algebraic strong shift equivalence for matrices over the integers, the proof requires a minor additional step (provided in [W3], following [Ef]). Effros [Ef] gave a related argument that algebraic shift equivalence implies algebraic strong shift equivalence for matrices over $S=\mathbf{Z}$. Both Williams' and Effros' proof work for $S$ a principal ideal domain.

Let $S$ be a commutative unital ring, let $\mathscr{P}$ denote the category of finitely generated projective modules, with $S$-module homomorphisms as maps, and let $\mathscr{F}$ denote the subcategory consisting of finitely generated free modules. In general, the Effros-Williams arguments that algebraic shift equivalence implies algebraic strong shift equivalence fails for morphisms in $\mathscr{F}$ (that is, matrices over $S$ ); for example, Proposition 2.1 rules out the use of a key step in Effros' proof. However, if $S$ is a Dedekind domain, the arguments work for morphisms in $\mathscr{P}$. This motivates the following easy, in fact trivial, excision theorem. (For background on projective modules over Dedekind domains, see [M, pp. 9-11].)

Lemma 2.3. If $P$ and $P^{\prime}$ are free finitely generated modules and $(\alpha, P)$ is algebraically strongly shift equivalent (algebraically shift equivalent) with respect to $\mathscr{P}$ to $\left(\beta, P^{\prime}\right)$, then they are algebraically strongly shift equivalent (algebraically shift equivalent) with respect to $\mathscr{F}$.

Proof. We prove the statement for algebraic strong shift equivalence; the argument for algebraic shift equivalence is even easier. Let $P_{1}$ be free, and $P_{2}$ and $P_{3}$ be projective, and let $\gamma_{i}, \delta_{i}(i=1,2)$ implement lag 1 algebraic strong shift equivalences with respect to $\mathscr{P}$ between $\left(\alpha_{i}, P_{i}\right)$ and $\left(\alpha_{i+1}, P_{i+1}\right)$. Choose a finitely generated projective module $Q_{2}$ such that $F_{2}=P_{2} \oplus Q_{2}$ is free. Define $\alpha^{\prime}=\alpha_{2} \oplus 0_{Q_{2}} \in$ End $F_{2}$, and set $\gamma_{1}^{\prime}$ to be the composition of $\gamma_{1}: P_{1} \rightarrow P_{2}$ with the inclusion of $P_{2}$ in $F_{2}$. Define $\delta_{1}^{\prime}: P_{2} \oplus Q_{2} \rightarrow P_{1}$ to be $\delta_{1}$ on $P_{2}$ and zero on $Q_{2}$. This yields an algebraic strong shift equivalence (with respect to $\mathscr{F}$ ) between $\left(\alpha_{1}, P_{1}\right)$ and $\left(\alpha_{2}^{\prime}, F_{2}\right)$. Now the algebraic strong shift equivalence between $\left(\alpha_{2}, P_{2}\right)$ and $\left(\alpha_{3}, P_{3}\right)$ can be extended to one between $\left(\alpha_{2}^{\prime}, F_{2}\right)$ and $\left(\alpha_{3}, P_{3}\right)$, and the process may be iterated.

An integral domain is called Prüfer if every finitely generated submodule of a free module is projective. Examples include Dedekind domains, as well as the ring of all algebraic integers (or any direct limit of Dedekind domains). Of course, Dedekind is equivalent to Prüfer and noetherian.

Now we are in a position to use Effros' argument.

Proposition 2.4. If $S$ is a Prüfer domain, algebraic shift equivalence implies algebraic strong shift equivalence.

Proof. By the preceding and the definitions, it is sufficient to show algebraic shift equivalence with respect to $\mathscr{P}$ implies algebraic strong shift equivalence with respect to $\mathscr{P}$. The following argument will show that if $\alpha$ is not nilpotent, we 
can reduce it to being one-to-one, while if it is nilpotent, $(\alpha, P)$ is algebraically strongly shift equivalent to the zero endomorphism (all zero endomorphisms are mutually algebraically strongly shift equivalent). If $n$ is the rank of $P$ (the dimension of the vector space $P \otimes_{S} K$, where $K$ is the field of fractions of $S$ ), $\operatorname{ker} \alpha^{n}=\operatorname{ker} \alpha^{n+1}=\cdots$. Hence $\alpha$ restricted to $\alpha^{n} P$ is one-to-one.

With $j: \alpha P \rightarrow P$ as the inclusion, the diagram

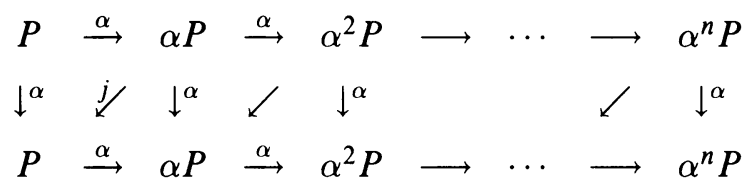

commutes. Reading off the columns, we see that $\left(\alpha \mid \alpha^{i} P, \alpha^{i} P\right)$ is algebraically strongly shift equivalent to $(\alpha, P)$. Each $\alpha^{i} P$ is projective, since $S$ is Prüfer.

Now suppose that $(\alpha, P)$ is algebraically shift equivalent to $\left(\alpha^{\prime}, P^{\prime}\right)$; by the preceding, we may assume that both $\alpha$ and $\alpha^{\prime}$ are one-to-one. Let $\gamma: P \rightarrow P^{\prime}$ and $\delta: P^{\prime} \rightarrow P$ be the intertwining operators such that $\gamma \delta=\left(\alpha^{\prime}\right)^{l}$ and $\delta \gamma=\alpha^{l}$. Since $\alpha$ and $\alpha^{\prime}$ are one-to-one, so are $\gamma$ and $\delta$. Set $Q_{k}=\gamma P+\left(\alpha^{\prime}\right)^{k} P^{\prime} \subseteq P^{\prime}$, for $k=0,1, \ldots, l$. Since $P$ and $P^{\prime}$ are finitely generated, so is $P_{k}^{\prime}$, and thus it is projective. Note that $Q_{0}=P^{\prime}, Q_{l}=\gamma P\left(\right.$ since $\left.\left(\alpha^{\prime}\right)^{k} P=\gamma \delta P^{\prime} \subseteq \gamma P\right)$, and $Q_{l} \subseteq Q_{l-1} \subseteq \cdots \subseteq Q_{0}$. Each $Q_{k}$ is $\alpha^{\prime}$-invariant (since $\alpha^{\prime} \gamma(P)=\gamma \alpha(P) \subseteq \gamma P$ ), and moreover $\alpha^{\prime} Q_{k} \subseteq Q_{k+1}$. Now the diagram

$$
\begin{array}{lllllll}
Q_{l} & \subseteq & Q_{l-1} & \subseteq & \cdots & \subseteq & P^{\prime} \\
\downarrow^{\alpha^{\prime}} & \downarrow_{\alpha^{\prime}} & \downarrow^{\alpha^{\prime}} & & & & \\
\alpha^{\prime} & \downarrow^{\alpha^{\prime}} \\
Q_{l} & \subseteq & Q_{l-1} & \subseteq & \cdots & \subseteq & P^{\prime}
\end{array}
$$

describes a chain of lag 1 algebraic strong shift equivalences, yielding that $\left(\alpha^{\prime}, Q_{l}\right)$ is algebraically strongly shift equivalent to $\left(\alpha^{\prime}, P^{\prime}\right)$. Now $\gamma$ is one-toone, so $\gamma: P \rightarrow \gamma P$ is an isomorphism, and thus $(\alpha, P)$ is algebraically strongly shift equivalent to $\left(\alpha^{\prime}, Q_{l}\right)$, and thus to $\left(\alpha^{\prime}, P^{\prime}\right)$.

To obtain results for more general rings, one requires a stronger excision result. For example, if $S$ is noetherian and has projective dimension $d$ (the case of $d=1$ is that of Dedekind domains), let $\mathscr{D}_{i}$ be the category of submodules of free modules that are finitely generated and have projective dimension $i-1$ or less. So $\mathscr{D}_{1}=\mathscr{P}$, for example. Then there should be an excision result asserting that if $P$ and $P^{\prime}$ belong to $\mathscr{D}_{i-1}$ but $(\alpha, P)$ and $\left(\alpha^{\prime}, P^{\prime}\right)$ are algebraically strongly shift equivalent or algebraically shift equivalent with respect to $\mathscr{D}_{i}$, then they should be equivalent with respect to $\mathscr{D}_{i-1}$. In particular, if such a result held, algebraic shift equivalence would imply algebraic strong shift equivalence for polynomial and Laurent polynomial rings in several variables over a field or a principal ideal domain.

Despite the algebraic nature of the proof of Proposition 2.4, we are unable to rule out, for every unital subring of $\mathbf{R}$, the possibility that algebraic shift equivalence does not imply algebraic strong shift equivalence. As a possible counterexample, let $S=\mathbf{Z}[2 \sqrt{2}]$; of course this does not have finite projective dimension. Let $A$ be the primitive matrix

$$
\left[\begin{array}{cc}
2 & 2 \sqrt{2} \\
2 \sqrt{2} & 4
\end{array}\right]
$$


Over the larger ring $\mathbf{Z}[\sqrt{2}], A$ is lag 1 strongly shift equivalent to the $1 \times 1$ matrix [6]. We do not know, however, if it is strongly shift equivalent or even algebraically strongly shift equivalent to [6] over $S$. Shift equivalence can be realized over $S$.

As a general remark, algebraic shift equivalence always carries with it complete information on $\mathrm{K}_{0}$; if $P$ and $Q$ are finitely generated projective modules, then $P \simeq Q$ if and only if $\left(i_{P}, P\right)$ is algebraically shift equivalent or algebraically strongly shift equivalent to $\left(i_{Q}, Q\right)$, where $i$ denotes the identity isomorphism (add a direct summand to make the module(s) free, as in the proof of Lemma 2.3). More generally, one can develop a Grothendieck group, even a ring structure, on the algebraic shift equivalence classes (as usual, this will entail a loss of information, since cancellation does not usually hold). This is related to $\mathrm{K}_{0}($ End $\mathscr{P})$ (see e.g., [Al, Ba]).

Lemma 2.5. Let $p_{1}(t)$ be a monic polynomial with coefficients from a Dedekind domain $S$ with nonzero constant term. Let $A$ be an $n \times n$ matrix with entries from $S$ whose characteristic polynomial is $p_{1}(t) p_{2}(t)$. Then $A$ is algebraically strongly shift equivalent over $S$ to a matrix of the form

$$
A^{\prime}=\left[\begin{array}{cc}
A_{11} & * \\
\mathbf{0} & A_{22}
\end{array}\right]
$$

such that for $i=1,2$, the characteristic polynomial of $A_{i i}$ is $p_{i}(t)$ or $t p_{i}(t)$.

Proof. Let $V=\operatorname{ker} p(A)$; this is clearly an $A$-invariant submodule of $S^{n}$, and since the image of $p(A)$ is projective (since it is a submodule of a free module and $S$ is Dedekind), $V$ is a direct summand of $S^{n}$. Write $S^{n}=V \oplus W$. If $V$ is free, then so is $W$ and we obtain the desired block upper triangularization at size $n$. If $V$ is not free, there is an ideal $I$ such that $F_{1}=V \oplus I$ is free; necessarily, $F_{2}=W \oplus I^{-1}$ is also free, and clearly $F_{1} \oplus F_{2}$ is free of rank $n+2$. Define $A^{\prime}: S^{n+2} \rightarrow S^{n+2}$ by letting it act as $A$ on $V$ and $W$, and as zero on the copies of $I$ and $I^{-1}$. Clearly $A^{\prime}$ leaves $F_{1}$ invariant, so with respect to the obvious choice of basis, it has the desired upper triangular form. Change of basis is implemented by conjugation by an invertible matrix and is thus an algebraic strong shift equivalence. It is immediate that $A$ and $A^{\prime}$ are algebraically strongly shift equivalent (see the proof of Lemma 2.3).

On the other hand, if $V$ is free, then so is $W$ and we obtain the desired block upper triangularization at size $n$.

Proposition 2.6. Let $A$ be a square matrix over a Dedekind domain $S$. Let $p(t)=t^{m} \prod_{i=1}^{k} p_{i}(t)$ be a factorization in $S[t]$ of the characteristic polynomial of $A$, where each $p_{i}$ is a monic polynomial with nonzero constant coefficient. Then $A$ is algebraically strongly shift equivalent over $S$ to a block matrix of the form

$$
A^{\prime}=\left[\begin{array}{llll}
A_{11} & & * & \\
& A_{22} & & \\
& & \ddots & \\
& & & A_{k k}
\end{array}\right],
$$

where $A_{i i} p_{i}\left(A_{i i}\right)=\mathbf{0}$ for $1 \leq i \leq k$.

Proof. The only problem with applying the preceding result inductively (with Lemma 1.3) is that a nilpotent part (corresponding to $t^{m}$ ) appears to have 
been left out. However, it follows from the proof of Proposition 2.4 that, over a Dedekind domain, any nilpotent endomorphism is algebraically strongly shift equivalent to the zero endomorphism.

Corollary 2.7. Let $S$ be a unital subring of $\mathbf{R}$ that is Dedekind. Let $A$ be a matrix with entries from $S$ and suppose that all of its eigenvalues belong to $S$. Then $A$ is algebraically strongly shift equivalent over $S$ to a block upper triangular matrix

$$
\left[\begin{array}{llll}
A_{11} & & * & \\
& A_{22} & & \\
& & \ddots & \\
& & & A_{k k}
\end{array}\right]
$$

such that for every $i, A_{i i}$ is either of size 2 or size 1, it has only one nonzero eigenvalue, and is either primitive or the negative of a primitive matrix.

Proof. If $S$ is $\mathbf{Z}$ (or any principal ideal domain), the blocks can be taken to be of size 1 , so the primitivity remark is obvious. Otherwise, we may assume $S$ is nondiscrete. Apply Proposition 2.6 to the polynomial $\prod\left(t-s_{i}\right)$; the diagonal block matrices that result are of size 1 or 2 ; if of size one, the matrix is simply [ $s_{i}$ ] so either it or its negative is primitive. If the size is 2 , by $[\mathrm{H} 1,2.2]$ (and the fact that powers of the matrix are multiples of it), we find a matrix $U$ in $\operatorname{SL}(2, \mathbf{R})$ so that one of $\pm U A_{i i} U^{-1}$ is strictly positive. Density of $\operatorname{SL}(2, S)$ in $\operatorname{SL}(2, \mathbf{R})$ yields $V$ in $\operatorname{SL}(2, S)$ so that one of $\pm V_{i} A_{i i} V_{i}^{-1}$ is strictly positive, hence primitive. Obviously conjugation with $\bigoplus V_{i}$ puts the matrix in the desired form.

\section{REALIZING ALgEBRAIC SHIFT EQUIVALENCE CLASSES BY PRIMITIVE MATRICES}

In this section, $S$ will always denote a unital subring of $\mathbf{R}$. Suppose that $A$ and $B$ are square matrices all of whose entries are nonnegative elements of $S$. We say that $B$ is embeddable in $A$ (over $S$ ), denoted $B \hookrightarrow A$, if $A$ is strongly shift equivalent (over $S_{+}$) to a matrix $A^{\prime}$ in which $B$ appears as a proper principal submatrix. In the trivial case that $A=[1]$ and $S=\mathbf{Z}$, no matrix $B$ is embeddable in $A$. In all other cases, the Submatrix Theorem [BH, 1.10 and 3.1] gives the following necessary and sufficient conditions for $B$ to be embeddable in $A$, when $A$ is primitive:

(i) The spectral radius of $A$ strictly exceeds that of $B$,

(ii) If $S$ is not discrete, for all positive integers $n$ and $k$,

$$
\left\{\begin{array}{l}
\operatorname{tr} B^{n} \leq \operatorname{tr} A^{n}, \\
\operatorname{tr} B^{n k}<\operatorname{tr} A^{n k} \quad \text { if } \operatorname{tr} B^{n}<\operatorname{tr} A^{n} .
\end{array}\right.
$$

(ii') If $S$ is discrete, then for all positive integers $n, \operatorname{tr}_{n} B \leq \operatorname{tr}_{n} A$.

If $A$ is a square matrix, we define the labelled graph $G_{A}$ whose vertices are the states, and there is an arc from $i$ to $j$ if and only if the $i, j$ entry of $A$ (denoted $A(i, j)$ ) is not zero. The arc is also labelled $A(i, j)$ in this case.

Let $N$ be a (square) matrix with $N^{l}=0$, let $F$ be another square matrix, and $W$ a rectangular matrix. Then $F$ is algebraically shift equivalent to $\left[\begin{array}{ll}N & W \\ 0 & F\end{array}\right]$ 
via the matrices $\left[\begin{array}{c}Z \\ F^{l}\end{array}\right]$ and $[\mathbf{0} \mathbf{I}]$, where $Z$ is the $(1,2)$ block entry of $\left[\begin{array}{ll}N & W \\ \mathbf{0} & F\end{array}\right]^{l}$. Plugging this algebraic shift equivalence into the Blocking Lemma (1.3), we deduce the algebraic shift equivalence of

$$
\left[\begin{array}{cc}
{\left[\begin{array}{cc}
B & C \\
D & |F|
\end{array}\right]} & {\left[\begin{array}{cc}
X_{1} & X_{2} \\
Y_{1} & Y_{2}
\end{array}\right]} \\
{\left[\begin{array}{ll}
\mathbf{0} & \mathbf{0} \\
\mathbf{0} & \mathbf{0}
\end{array}\right]} & {\left[\begin{array}{cc}
N & W \\
\mathbf{0} & F
\end{array}\right]}
\end{array}\right] \text { and }\left[\begin{array}{cc}
{\left[\begin{array}{cc}
B & C \\
D & |F|
\end{array}\right]} & \left.\left[\begin{array}{c}
X_{1} Z+X_{2} F^{l} \\
Y_{2} Z+Y_{2} F^{l}
\end{array}\right]\right] \\
{\left[\begin{array}{l}
\mathbf{0}
\end{array}\right]} & F
\end{array}\right]
$$

For future reference, we note that

$$
Z=N^{l-1} W+N^{l-2} W F+\cdots+N W F^{l-2}+W F^{l-1} .
$$

We are now ready to prove our central realization result. Below if $F$ is a matrix, then $|F|$ denotes the "absolute value of $F$," given by $|F|(i, j)=$ $|F(i, j)|$.

Theorem 3.1. Let $S$ be a unital subring of $\mathbf{R}$. Assume that either $S=\mathbf{Z}$ or that $S$ has a nontrivial unit. Suppose that $\bar{M}=\left[\begin{array}{l}A J \\ \mathbf{0} F\end{array}\right]$ is a square matrix over $S$ such that $A$ is primitive and $|F| \hookrightarrow A$. Then $\bar{M}$ is algebraically shift equivalent (over $S$ ) to a primitive matrix.

Remark. We believe that the requirement of the existence of a nontrivial unit is an artifact of our proof. The result is still quite general-for example, if $S$ contains an irrational algebraic number or a rational that is not an integer, then $S$ has a nontrivial unit.

Proof. Fix $\varepsilon$ in $S$ with $0<\varepsilon \leq \lambda(|F|)$. If $S=\mathbf{Z}$, let $\varepsilon=1$. First, we reduce to the case that $|F|$ is primitive. By the Blocking Lemma (1.3), it is enough to produce a matrix $F^{\prime}$ algebraically shift equivalent to $F$ (over $S$ ), such that $\left|F^{\prime}\right|$ is primitive and $\left|F^{\prime}\right| \hookrightarrow A$. For an integer $l$, let $Q_{l}$ be the nilpotent matrix of size $l$ given by

$$
Q_{l}(i, j)= \begin{cases}1 & \text { if } j=i+1 \\ 0 & \text { otherwise }\end{cases}
$$

Define

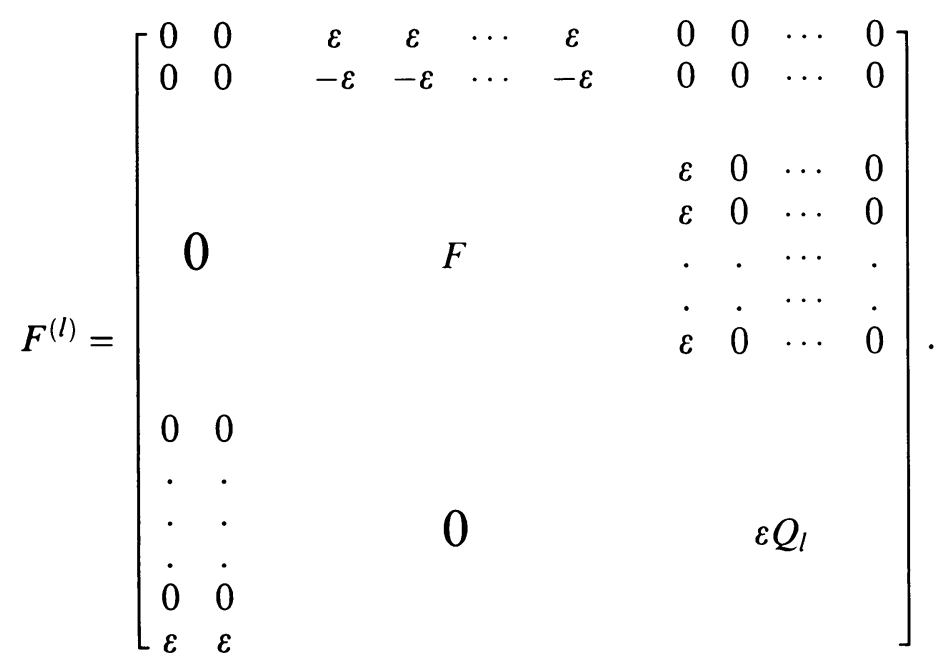


Then $F^{(l)}$ is algebraically shift equivalent to $F$ (over $S$ ); to see this, amalgamate the first two rows and ignore the nilpotent block. Moreover, $\left|F^{(l)}\right|$ is primitive. For large $l,\left|F^{(l)}\right| \hookrightarrow A$. Hence we may assume $|F|$ is primitive.

By the Submatrix Theorem and the Blocking Lemma (1.3), we may suppose (on passing to an $S_{+}$-strongly shift equivalent matrix) that for some sufficiently large integer $k, A$ is in the block form:

$$
A=\left[\begin{array}{cccc}
* & * & * & * \\
* & \varepsilon Q_{k} & E & 0 \\
* & 0 & B & C \\
* & D & 0 & |F|
\end{array}\right],
$$

where:

- $B$ is primitive;

- $\lambda(B)>\lambda(|F|)$;

- no entry of $B$ has modulus exceeding 1 ;

- $C$ is the matrix all of whose entries are 0 , except along the bottom row, where they are $\varepsilon$;

- $D$ is the matrix with all entries in the leftmost column equal to $\varepsilon$, and all others consisting of zero;

- $E$ has its unique nonzero entry, equalling 1 , in the lower left corner.

To achieve all this, we may choose $B$ to satisfy the first three conditions and so that in addition

$$
\left[\begin{array}{cc}
B & C \\
0 & |F|
\end{array}\right] \hookrightarrow A .
$$

Then choose $k$ large enough that

$$
\left[\begin{array}{ccc}
\varepsilon Q_{k} & E & 0 \\
0 & B & C \\
D & 0 & |F|
\end{array}\right]
$$

also embeds in $A$ (using the Submatrix Theorem).

If $S$ is not the integers, by assumption it must contain an arbitrarily large unit. For any matrix with entries from $S, M$, and positive unit $u$ in $S, M$ is algebraically shift equivalent over $S$ to a primitive matrix if and only if $u M$ is. If $S$ is not equal to $\mathbf{Z}$, we multiply the matrix $\left[\begin{array}{ll}A & J \\ 0 & F\end{array}\right]$ by a large unit; this permits us to assume that every nonzero entry of $A$ exceeds 1 .

By Corollary 1.9, we may choose a strictly positive matrix $H$ with entries from $S$ such that for any integer $k$,

$$
\left[\begin{array}{cc}
A & J \\
0 & F
\end{array}\right] \quad \text { and } \quad\left[\begin{array}{cc}
A & J+k H \\
0 & F
\end{array}\right]
$$

are algebraically shift equivalent over $S$. In particular, we may suppose that $J$ is strictly positive.

Let $\mathscr{I}$ be an index set for the rows and columns of $A$, and let $\mathcal{J}$ be an index set for the rows and columns of $F$. Define a matrix $\tilde{A}$ from $A$ by splitting the bottom block row according to the decomposition

$$
\left[\begin{array}{llll}
* & D & \mathbf{0} & |F|
\end{array}\right]=\left[\begin{array}{llll}
\mathbf{0} & \mathbf{0} & \mathbf{0} & |F|
\end{array}\right]+\left[\begin{array}{llll}
* & D & \mathbf{0} & \mathbf{0}
\end{array}\right] .
$$


Now $\tilde{A}$ has the block form

$$
\begin{aligned}
\widetilde{A} & =\left[\begin{array}{ccccc}
* & * & * & * & * \\
* & \varepsilon Q_{k} & E & \mathbf{0} & \mathbf{0} \\
* & \mathbf{0} & B & C & C \\
* & D & \mathbf{0} & \mathbf{0} & \mathbf{0} \\
& & & & \\
\mathbf{0} & \mathbf{0} & \mathbf{0} & |F| & |F|
\end{array}\right] \\
& =\left[\begin{array}{ccc}
\widetilde{A_{1}} & * \\
* & |F|
\end{array}\right] .
\end{aligned}
$$

Now let $\mathscr{I} \dot{\cup} \mathscr{J}$ index the rows of $\tilde{A}$. The first four block rows are indexed by $\mathscr{I}$ in the natural way. The rows that contain the rows of $|F|$ are indexed by $\mathcal{J}$, corresponding to the manner in which $\mathcal{J}$ indexes the rows of $F$. The forms of $C$ and $D$ and the primitivity of $A_{1}$ guarantee that $\widetilde{A_{1}}$ is primitive. The spectral radius of $\tilde{A}$ exceeds $\lambda_{|F|}$, since $\lambda_{B}>\lambda_{|F|}$.

Let $\tilde{A}=\widetilde{A}^{(0)}=\left[\begin{array}{ll}R^{(0)} & S^{(0)} \\ T^{(0)} & |F|\end{array}\right]$. For $m \geq 0$, given $\widetilde{A}^{(m)}=\left[\begin{array}{ll}R^{(m)} & S^{(m)} \\ T^{(m)} & |F|\end{array}\right]$ with $\mathscr{I}(m)$ indexing the rows of $\left[\begin{array}{ll}R^{(m)} & S^{(m)}\end{array}\right]$, and $\mathscr{J}$ indexing the rows and columns of $|F|$, we define

$$
\widetilde{A}^{(m+1)}=\left[\begin{array}{cc}
R^{(m+1)} & S^{(m+1)} \\
T^{(m+1)} & |F|
\end{array}\right]
$$

by completely splitting the rows of $\widetilde{A}^{(m)}$ indexed by $\mathscr{I}(m)$. That is, the row splitting amounts to writing (for each $\alpha$ in $\mathscr{I}(m)$ ) row $\alpha$ as the sum of orthogonal rows, each of which has precisely one nonzero entry. Explicitly, $\alpha$ will be a string of symbols and the row in $\widetilde{A}^{(m+1)}$ corresponding to $\widetilde{A}^{(m)}\left(\alpha, \alpha^{\prime}\right) \neq 0$ will be indexed by a string $\beta$, defined as follows:

- If the terminal symbol of $\alpha$ is in $\mathscr{J}$, then $\beta=\alpha$;

- otherwise, $\beta=\alpha k$, where $k$ is the terminal symbol of $\alpha^{\prime}$.

This defines $\mathscr{I}(m+1)$ from $\mathscr{I}(m)$.

So $\mathscr{I}(m)$ is the union of paths of vertices, $i_{1} i_{2} \cdots i_{m+1}$ from $G_{\widetilde{A}_{1}}$, together with the set

$$
\begin{aligned}
& \widetilde{\mathcal{I}}^{(m)}=\left\{i_{1} i_{2} \cdots i_{t} j \mid 1 \leq t \leq m ; i_{1} i_{2} \cdots i_{t} \text { is a path of vertices in } G_{\widetilde{A}_{1}}\right. \\
& \left.\qquad j \in \mathcal{J} ; \widetilde{A}\left(i_{t}, j\right)>0\right\} .
\end{aligned}
$$

Now consider a matrix of the form $\left[\begin{array}{cc}\widetilde{A}^{(m)} & K \\ 0 & F\end{array}\right]$. This is shift equivalent to $\left[\begin{array}{ll}A & \bar{K} \\ 0 & F\end{array}\right]$, obtained by successively amalgamating rows not corresponding to $F$. Here $\bar{K}(i, j)$ is the sum of all the terms $K(w, j)$ such that $w$ is an index which ultimately amalgamates to $i$. When the string $w$ does not belong to $\mathscr{J}$, it amalgamates to $i$ if $i$ is the initial symbol of $w$. If $w$ does belong to $\mathscr{J}$, then $w$ amalgamates to the unique symbol $i$ from which it was split in the creation of $\widetilde{A}$ from $A$.

We will construct a matrix $\widetilde{M}$ that is algebraically shift equivalent over $S$ to a primitive matrix that is also algebraically shift equivalent over $S$ to a matrix of the form

$$
\left[\begin{array}{cc}
\widetilde{A}^{(m)} & \bar{K} \\
\mathbf{0} & F
\end{array}\right]
$$


with $\bar{K}=J+n H$ for some integer $n$. This will finish the proof.

We will choose (after a suitable ordering of the rows through $R^{(m)}$ in $\widetilde{A}^{(m)}$ ) $\widetilde{M}$ to have the block form

$$
\widetilde{M}=\left[\begin{array}{cc}
\widetilde{A}^{(m)} & * \\
\mathbf{0} & *
\end{array}\right]=\left[\begin{array}{ccccc}
B_{1} & B_{2} & B_{3} & \mathbf{0} & \mathbf{0} \\
B_{4} & B_{5} & B_{6} & X & \mathbf{0} \\
B_{7} & B_{8} & |F| & \mathbf{0} & F_{-} \\
& & & & \\
\mathbf{0} & \mathbf{0} & \mathbf{0} & N & W \\
\mathbf{0} & \mathbf{0} & \mathbf{0} & \mathbf{0} & F
\end{array}\right]
$$

Here $R^{(m)}=\left[\begin{array}{ll}B_{1} & B_{2} \\ B_{4} & B_{5}\end{array}\right]$, and $|F|=F_{+}+F_{-} ; F=F_{+}-F_{-} ; F_{+}, F_{-} \geq \mathbf{0} ;$ and $X$ has size matching that of $N$; moreover, $N$ will be a nilpotent $0-1$ matrix in order to force the algebraic shift equivalence of $\left[\begin{array}{ll}N & W \\ 0 & F\end{array}\right]$ to $F$.

The matrix $M$ is obtained by adding the block rows 2 and 3 to the block rows 4 and 5 respectively, and then subtracting block columns 4 and 5 from block columns 2 and 3 . This yields

$$
M=\left[\begin{array}{ccccc}
B_{1} & B_{2} & B_{3} & \mathbf{0} & \mathbf{0} \\
B_{4} & B_{5}-X & B_{6} & X & \mathbf{0} \\
B_{7} & B_{8} & F_{+} & \mathbf{0} & F_{-} \\
& & & & \\
B_{4} & B_{5}-X-N & B_{6}-W & X+N & W \\
B_{7} & B_{8} & F_{-} & \mathbf{0} & F_{+}
\end{array}\right]=\left[\begin{array}{ll}
M_{1} & M_{2} \\
M_{4} & M_{5}
\end{array}\right] .
$$

We now describe general forms for our choices of $X, N$, and $W$.

We select a certain subset $\mathscr{W}^{(m)}=\mathscr{W}$ of words from $\widetilde{\mathcal{I}}^{(m)}$ of the form $i_{1} \cdots i_{m} j$. Let $\overline{\mathscr{W}}$ denote the set of terminal segments of length 2 or more of the words of $\mathscr{W}$. We group the rows of $\widetilde{A}^{(m)}$ so that $\mathscr{W}$ indexes the rows through $B_{5}$. We also let $\mathscr{W}$ index the rows and columns of $X$ and $N$. Then we set all entries of $X$ equal to zero except that for each word $i_{1} \cdots i_{m} j$ in $\mathscr{W}$, we require

$$
0<X\left(i_{1} \cdots i_{m} j, i_{2} \cdots i_{m} j\right) \leq B_{5}\left(i_{1} \cdots i_{m} j, i_{2} \cdots i_{m} j\right) .
$$

Set every entry of $N$ equal to zero except that for the words in $\mathscr{W}$ of the form $i_{1} \cdots i_{t} j, i_{2} \cdots i_{t} j$ with $1<t<m$, we require

$$
0<N\left(i_{1} \cdots i_{t} j, i_{2} \cdots i_{t} j\right) \leq B_{5}\left(i_{1} \cdots i_{t} j, i_{2} \cdots i_{t} j\right) .
$$

Index the rows and columns of $W$ by $\mathscr{W}$ and $\mathscr{J}$, respectively. Finally, we require the entries of $W$ to be zero except that $0<W(i j, j) \leq B_{6}(i j, j)$ for every word $i j$ of length 2 in $\overline{\mathscr{W}}$. With these choices, the matrix $M$ will be nonnegative. It is not difficult to verify that it must then be primitive.

By the remarks preceding the statement of this theorem, $\widetilde{M}$ is algebraically shift equivalent over $S$ to the matrix

$$
\left[\begin{array}{cccc}
B_{1} & B_{2} & B_{3} & \mathbf{0} \\
B_{4} & B_{5} & B_{6} & X Z \\
B_{7} & B_{8} & |F| & F_{-} F^{l} \\
\mathbf{0} & \mathbf{0} & \mathbf{0} & F
\end{array}\right]
$$


where $l=m-1, N^{l}=\mathbf{0}$, and $Z=N^{l-1} W+N^{l-2} W F+\cdots+N W F^{l-2}+$ $W F^{l-1}$. A consequence of the last equation is that $X Z=X N^{l-1} W$, so that all the entries of $X Z$ are zero, except the $\left(i_{1} \cdots i_{m} j, j\right)$ entries, given by words $i_{1} \cdots i_{m} j$ in $\mathscr{W}$. Such an entry can be as large as

$$
\left(B_{5}\right)^{m-1}\left(i_{1} \cdots i_{m} j, j\right) B_{6}\left(i_{m} j, j\right),
$$

depending on our selections for entries in $X, N$, and $W$.

Thus we will be done if we can make our choices of $m$ (for $X^{(m)}$ ), $\mathscr{W}$, $X, N$, and $W$ such that for some integer $r$ with $\left[\begin{array}{cc}\widetilde{A}^{(m)} & K^{(m)} \\ 0 & F\end{array}\right]$ given by

$$
K^{(m)}=\left[\begin{array}{c}
\mathbf{0} \\
X Z \\
F_{-} F^{m-1}
\end{array}\right]
$$

we have $\bar{K}=J+r H$.

The contribution of $\left|F_{-} F^{m-1}\right|$ to any entry of $\bar{K}$ is $O\left(\lambda_{|F|}^{m}\right)$. On the other hand, for each $i$, the maximum number of words in $\mathscr{W}^{(m)}$ beginning with $i$ grows exponentially faster than $\lambda_{|F|}^{m}$. In particular, with $S=\mathbf{Z}$, for sufficiently large $m$, we simply choose an appropriate number of such words for each $i$ so that $\bar{K}$ has the desired form (on setting the nonzero entries of $X, N$, and $W$ to be one).

If $S \neq \mathbf{Z}$, then for sufficiently large $m$, we still have that the maximum contribution of $X Z$ to any entry of $\bar{K}$ is large compared to contributions from $F_{-} F^{l}, J$, and $H$. Thus given $\varepsilon>0$, we can arrange that every entry of $\bar{K}$ be within $\varepsilon$ of $J+r H$, with flexibility remaining in the choices for $X$, $Z$, and $W$. As every nonzero entry of $A$ is larger than 1 and this condition persists after our splittings to $\widetilde{A}^{(m)}$, we can cover a small error term $s$ by an additional entry of $X N^{l-1} W$ corresponding to a product $1 \cdot 1 \cdots s$.

Lemma 3.2. Let $\lambda$ be a real number, and let $n$ be a positive integer. Then

$$
\operatorname{tr}_{n}(-\lambda)= \begin{cases}-\operatorname{tr}_{n}(\lambda) & \text { if } n \text { is odd, } \\ \operatorname{tr}_{n}(\lambda) & \text { if } n \equiv 0 \text { mod } 4 \\ \operatorname{tr}_{n}(\lambda)+2 \operatorname{tr}_{q}(\lambda) & \text { if } n=2 q \text { with } q \text { odd. }\end{cases}
$$

Proof. If $n$ is odd, the result is clear; if $n$ is divisible by 4 , the Möbius function ignores all the quotients $n / d$ where $d$ is odd, and again the result follows. The third statement is a routine calculation.

Theorem 3.3. Let $S$ be a unital subring of $\mathbf{R}$. Suppose that either $S=\mathbf{Z}$ or $S$ is a Dedekind domain with a nontrivial unit. Let $A$ be a square matrix with entries from $S$ whose nonzero spectrum $\Delta$ satisfies the necessary conditions of the Spectral Conjecture and consists of elements of $S$. Then $A$ is algebraically shift equivalent over $S$ to a primitive matrix.

Proof. Arrange the elements of $\Delta$ in decreasing order, $\Delta=\left(\lambda_{1}, \ldots, \lambda_{k}\right)$. We must first show that initial segments of $\Delta$ also satisfy the conditions of the Spectral Conjecture, and that $\left[\left|\lambda_{s}\right|\right] \hookrightarrow C$ if $C$ is a matrix with nonzero spectrum $\Delta^{\prime}=\left(\lambda_{1}, \ldots, \lambda_{s-1}\right)$. If $\lambda_{s}>0$, the spectral conditions are automatically satisfied by $\Delta^{\prime}$, so we may assume $\lambda_{s}<0$. If $n$ is even, $\operatorname{tr}\left(\Delta^{\prime}\right)^{n}>0$ automatically (since all the eigenvalues are real), and if $n$ is odd, $\operatorname{tr}\left(\Delta^{\prime}\right)^{n}>\operatorname{tr} \Delta^{n}$. If 
$S=\mathbf{Z}$, we must consider the $n$th net traces. We use Lemma 3.2. If $n$ is odd, $\operatorname{tr}_{n} \Delta^{\prime}>\operatorname{tr}_{n} \Delta$. If $n$ is even, $\operatorname{tr}_{n} \Delta^{\prime}>0$ in any case (since the eigenvalues are all real). We thus have that all initial segments of $\Delta$ also satisfy the conditions of the Spectral Conjecture.

In order to use Theorem 3.1, we are required to verify similar tracial conditions for embeddability. Explicitly, if $S=\mathbf{Z}$, we wish to show that $\operatorname{tr}_{n} \Delta^{\prime} \geq$ $\operatorname{tr}_{n}\left(\left|\lambda_{s}\right|\right)$. Again, we may suppose $\lambda_{s}<0$. If $n$ is odd,

$$
\operatorname{tr}_{n} \Delta^{\prime}-\operatorname{tr}_{n}\left(\left|\lambda_{s}\right|\right)=\operatorname{tr}_{n} \Delta^{\prime}-\operatorname{tr}_{n}\left(-\lambda_{s}\right)=\operatorname{tr}_{n} \Delta^{\prime}+\operatorname{tr}_{n}\left(\lambda_{s}\right)=\operatorname{tr}_{n} \Delta^{\prime \prime} \geq 0,
$$

where $\Delta^{\prime \prime}=\left(\lambda_{1}, \ldots, \lambda_{s}\right)$. If 4 divides $n$,

$$
\operatorname{tr}_{n} \Delta^{\prime}=\operatorname{tr}_{n}\left(\left|\Delta^{\prime}\right|\right) \geq \operatorname{tr}_{n}\left(\lambda_{1}\right) \geq \operatorname{tr}_{n}\left(\lambda_{s}\right) .
$$

If $n=2 q$ with $q$ odd, let $\lambda_{l}$ be the first negative element of $\Delta$. Then

$$
\begin{aligned}
\operatorname{tr}_{n} \Delta^{\prime} & =\sum_{i<l} \operatorname{tr}_{n}\left(\lambda_{i}\right)+\sum_{i=l}^{s-1} \operatorname{tr}_{n}\left(\lambda_{i}\right) \\
& =\sum_{i<l} \operatorname{tr}_{n}\left(\lambda_{i}\right)+\sum_{i=l}^{s-1}\left(\operatorname{tr}_{n}\left(\left|\lambda_{i}\right|\right)+2 \operatorname{tr}_{q}\left(\left|\lambda_{i}\right|\right)\right) \geq \operatorname{tr}_{n}\left(\lambda_{1}\right) \geq \operatorname{tr}_{n}\left(\left|\lambda_{s}\right|\right) .
\end{aligned}
$$

To verify the embeddability conditions over nondiscrete rings, we simply note that the qualitative tracial conditions (see (ii) in the beginning of this section) on $\Delta$ are equivalent to the corresponding conditions on the the net traces of $N \Delta$ for some sufficiently large $N$ [BH, Lemma 1.1].

Now suppose that $S$ is a principal ideal domain. By Theorem 1.4 and Proposition 1.5 , there exists a nonsingular triangular matrix $B$, algebraically shift equivalent over $S$ to $A$ with $B(i, i)=\lambda_{i}$ for $1 \leq i \leq s$. Let $B_{k}$ be the upper left $k \times k$ block of $B$, so $B$ has the form

$$
\left[\begin{array}{cc}
B_{k} & * \\
\mathbf{0} & C_{k}
\end{array}\right]
$$

for $1 \leq k \leq s$. Each subtuple $\left(\lambda_{1}, \ldots, \lambda_{k}\right)$ of $\Delta$ satisfies the necessary conditions of the spectral conjecture. In particular, by Theorem 3.1, $B_{2}$ is algebraically shift equivalent over $S$ to a primitive matrix $D_{2}$. By the Blocking Lemma (1.3), $B$ is algebraically shift equivalent to a matrix of the form

$$
\left[\begin{array}{cc}
D_{2} & * \\
\mathbf{0} & C_{2}
\end{array}\right] \text {. }
$$

The same argument permits us to pass to

$$
\left[\begin{array}{cc}
D_{3} & * \\
\mathbf{0} & C_{3}
\end{array}\right]
$$

with $D_{3}$ primitive, and so on until the primitive matrix $D_{s}$ is obtained; this is algebraically shift equivalent to $A$.

When $S$ is a nondiscrete Dedekind domain, there is an additional step. Instead of the triangular matrix $B$, by Corollary 2.7 , we have a block triangular matrix $\widetilde{B}$ where the $i$ th diagonal block is of size 2 with eigenvalues $\lambda_{i}$ and 0 , and either $\widetilde{B}_{i}$ or its negative is primitive. Now the proof goes through using the matrices $\widetilde{B}_{i}$ in place of the entries $\lambda_{i}$ used in the principal ideal domain situation. 
If $A$ is diagonable ${ }^{1}$ over $\mathbf{R}$ and its nonzero spectrum satisfies the hypotheses of the Spectral Conjecture, then, by Theorem 3.3, it is algebraically shift equivalent to a primitive matrix. Any symmetric primitive matrix is diagonable over R.

Question 3.4. Suppose that $\Delta$ has all of its entries real and satisfies the conditions of the Spectral Conjecture. Does there exist a symmetric primitive matrix with nonzero spectrum $\Delta$ ?

Lemma 3.5. Let $S$ be a nondiscrete subring of $\mathbf{R}$, and let $A$ be a square matrix with entries from $S$. Suppose that $\operatorname{tr} A>0$ and $A$ is algebraically shift equivalent over the reals to a primitive (real) matrix. Then $A$ is algebraically shift equivalent over $S$ to a strictly positive matrix.

Proof. Let $D$ be a primitive real matrix algebraically shift equivalent over $\mathbf{R}$ to $A$. By [KR2], there exists a strictly positive matrix $D_{1}$ that is algebraically shift equivalent (over $\mathbf{R}$ ) to $D$. Choose nilpotent zero-one matrices $N$ and $N^{\prime}$ such that $A \oplus N^{\prime}$ is conjugate over $\mathbf{R}$ to $D_{1} \oplus N$. By Theorem 1.10, $D_{1}$ is strongly shift equivalent over $S_{+}$to a strictly positive matrix $D_{2}$ which is conjugate over $\mathbf{R}$ to $D_{1} \oplus N$. Thus $D_{2}$ is conjugate over $\mathbf{R}$ to $A^{\prime}=A \oplus N^{\prime}$, and $A^{\prime}$ is algebraically shift equivalent over $S$ to $A$. As $S$ is dense in $\mathbf{R}, \operatorname{SL}(\cdot, S)$ is dense in $\operatorname{SL}(\cdot, \mathbf{R})$, and it follows as in [BH, proof of Theorem, Appendix 4], that there exists $P$ in $\mathrm{SL}(\cdot, S)$ such that $P A^{\prime} P^{-1}$ is strictly positive.

Corollary 3.6. Let $S$ be a dense subring of $\mathbf{R}$. Let $A$ be a square matrix over $S$ whose nonzero spectrum satisfies the conditions of the spectral conjecture; assume in addition that $\operatorname{tr} A>0$ and all eigenvalues of $A$ are real. Then $A$ is algebraically shift equivalent over $S$ to a strictly positive matrix.

Proof. Since $\mathbf{R}$ is obviously a principal ideal domain, we may apply Theorem 3.3; so $A$ is algebraically shift equivalent over $\mathbf{R}$ to a primitive matrix. Now Lemma 3.5 yields the result.

Let $A$ be a real square matrix whose nonzero spectrum is $\Delta=\left(\lambda_{1}, \ldots, \lambda_{s}\right)$. Set $\chi_{A}^{*}(t)=\prod_{i=1}^{s}\left(t-\lambda_{i}\right)$. Recall that $A$ is algebraically shift equivalent over $\mathbf{R}$ to a matrix whose characteristic polynomial is $\chi_{A}^{*}$, and $A$ is algebraically shift equivalent to the companion matrix for $\chi_{A}^{*}$ if and only if $\chi_{A}^{*}$ divides the minimal polynomial of $A$. This is a "robust" condition; in the space of matrices whose characteristic polynomial is that of $A$, the set of matrices algebraically shift equivalent to the companion matrix of $\chi_{A}^{*}$ is open, i.e., stable under perturbations, and the others are not. A sufficient condition for $\chi_{A}^{*}$ to divide the minimal polynomial of $A$ is that the characteristic polynomial of $A$ admit no repeated nonzero roots.

Theorem 3.7. Let $S$ be a dense unital subring of $\mathbf{R}$, and let $A$ be a square matrix with entries from $S$ whose nonzero spectrum satisfies the necessary conditions of the Spectral Conjecture. Assume in addition that

(i) $\operatorname{tr} A>0$,

(ii) $\chi_{A}^{*}$ divides the minimal polynomial of $A$.

Then $A$ is algebraically shift equivalent over $S$ to a strictly positive matrix.

Proof. By [BH, Theorem, Appendix 4], there exists an integer $e$ so that the companion matrix of $t^{e} \chi_{A}^{*}$ is conjugate over $\mathbf{R}$ to some strictly positive matrix

\footnotetext{
${ }^{1} \mathrm{~A}$ better word than the unpronounceable "diagonalizable".
} 
$B$. Over $\mathbf{R}, B$ is algebraically shift equivalent to the companion matrix of $\chi_{A}^{*}$. Hence $A$ is algebraically shift equivalent over $\mathbf{R}$ to a strictly positive matrix and Lemma 3.5 applies.

Let $S$ be a unital subring of $\mathbf{R}$. Let $p$ be a monic polynomial of positive degree and nonzero constant term with coefficients from $S$. Let $C$ be the companion matrix of $p$. Given $\varepsilon>0$, the Radius Theorem of [BH] constructs a matrix $A$ such that $\chi_{A}^{*}=p$ and the spectral radii of $|A|$ and $C$ differ by less than $\varepsilon$. (The theorem also provides controls on the entries of $A$ contributing to traces of small powers.) The matrix $A$ produced by the construction is algebraically shift equivalent over $S$ to $C$.

We could improve the spectral condition in the statement of Theorem 3.3 if we had a Radius Theorem which controlled the algebraic shift equivalence class.

Radius Conjecture 3.8. Suppose that $A$ is a square matrix over a unital subring $S$ of $\mathbf{R}$ and $\varepsilon>0$. Then there exists a matrix $B$ algebraically shift equivalent over $S$ to $A$, such that the spectral radii of $|B|$ and $A$ are within $\varepsilon$ of each other.

We also expect the matrix $B$ to come with control over entries contributing to traces of small powers as in [BH, Theorem 2.5].

\section{REALIZING TWO INTEGER EIGENVALUES}

In this section, we work over the integers and prove the following result by explicit constructions.

Theorem 4.1. Suppose a square integer matrix has exactly two nonzero integer eigenvalues $n$ and $k$ with multiplicity one and $n>|k|$. Then the matrix is algebraically shift equivalent over $\mathrm{Z}$ to a primitive matrix of size 4 or less.

The condition $n>|k|$ is obviously a necessary condition. Of course, by Theorem 3.3, we know that $A$ is algebraically shift equivalent to some primitive matrix. One motive behind Theorem 4.1 is to establish, at least for this first nontrivial situation, a sharp upper bound for the minimum size of the realizing primitive matrix (4, in this case). We also wish to exhibit the complications involved in explicit realization. Finally, we want to build a stockpile of examples for use in symbolic dynamics.

The proof of Theorem 4.1 proceeds by cases. By Proposition 1.7, every matrix with these as its nonzero eigenvalues is algebraically shift equivalent over $\mathbf{Z}$ to a matrix of the form $\left[\begin{array}{ll}n & x \\ 0 & k\end{array}\right]$, and we may assume that $x$ runs over a set of representatives modulo $n-k$.

Proposition 4.2. Assume $k>0$.

(a) If $0<x<n-k$, then $\left[\begin{array}{ll}n & x \\ 0 & k\end{array}\right]$ is $\mathrm{SL}(2, \mathbf{Z})$-conjugate to a strictly positive matrix.

(b) If $k$ and $n$ are not relatively prime, then $\operatorname{diag}(n, k)$ is algebraically shift equivalent to a strictly positive matrix.

Proof. (a) We use the conjugacy

$$
\left[\begin{array}{ll}
1 & 0 \\
1 & 1
\end{array}\right]\left[\begin{array}{ll}
n & x \\
0 & k
\end{array}\right]\left[\begin{array}{cc}
1 & 0 \\
-1 & 1
\end{array}\right]=\left[\begin{array}{cc}
n-x & x \\
n-k-x & x+k
\end{array}\right]>\mathbf{0}
$$


(b) As $n$ and $n-k$ have a common divisor exceeding 1 , there exists an integer $a$ with $1 \leq a \leq n-k-1$ such that $n a$ is divisible by $n-k$. By Proposition $1.7, \operatorname{diag}(n, k)$ is algebraically shift equivalent to $\left[\begin{array}{ll}n & a \\ 0 & k\end{array}\right]$; this is conjugate to a strictly positive matrix, by $(a)$.

For fixed $n$ and $k$, we say the integer $a$ is "attained" by a primitive matrix $C$ (or simply attained, if there is no reference to $C$ ) if $\left[\begin{array}{ll}n & a \\ 0 & k\end{array}\right]$ is algebraically shift equivalent to $C$.

Proposition 4.3. Let $t, u$, and $v$ be integers such that $0 \leq t, u, v$ $\leq n-1$. The matrices

$$
A=\left[\begin{array}{cc}
n & t+(n-1) u \\
0 & v-u
\end{array}\right], \quad B=\left[\begin{array}{ccc}
1 & n-1-t & t \\
1 & n-1-u & u \\
1 & n-1-v & v
\end{array}\right]
$$

are algebraically strongly shift equivalent over $\mathbf{Z}$.

(a) If $n-1>|k|$, then every algebraic shift equivalence class of $\left[\begin{array}{ll}n & x \\ 0 & k\end{array}\right]$ contains a primitive matrix of size 3 .

(b) If $k=n-1, x=0$, and $n \geq 3$, then $\operatorname{diag}(n, k)$ is algebraically shift equivalent to a primitive $3 \times 3$ matrix.

(c) An integer matrix $\left[\begin{array}{cc}n & x \\ 0 & 1-n\end{array}\right]$ is $\mathrm{SL}(2, \mathbf{Z})$-conjugate to one of the same form for which $0 \leq x \leq n$; all of these except possibly $\operatorname{diag}(2,1)$, $\operatorname{diag}(2 d, 1-2 d)$, and $\left[\begin{array}{cc}6 e+5 & 4 e+3 \\ 0 & -6 e-4\end{array}\right](d \geq 1$ and $e \geq 0)$, are algebraically shift equivalent to a primitive matrix of size 3.

Proof. There is a sequence of algebraic strong shift equivalences

$$
A \rightarrow\left[\begin{array}{ccc}
n & 0 & t+(n-1) u \\
1 & 0 & u \\
0 & 0 & v-u
\end{array}\right] \rightarrow\left[\begin{array}{ccc}
1 & n-1 & t \\
1 & n-1 & u \\
0 & 0 & v-u
\end{array}\right] \rightarrow B
$$

Deleting the second row and column from the second matrix yields $A$. Subtracting $(n-1)$ times the first column of the third matrix from the second column, and adding $n-1$ times the second row to the first yields the second matrix. Finally, $B$ is obtained from the third matrix by subtracting the third column from the second and adding the second row to the third.

(a) If $k>0$, set $u=1$ and $v=k-1$; we observe that each such choice forces $B$ to be primitive for $0 \leq t \leq n-2, t+(n-1) u$ ranges over $n-1$ consecutive values for $x$ (in the matrices of the form $A$. As $n-k \leq n-1$, all algebraic shift equivalence classes are obtained, by Proposition 1.7.

If $k<0$, set $v=0$ and $u=-k$; for $0 \leq t \leq n-2$, the corresponding $B$ 's are all primitive. The $(1,2)$-entry of $A$ runs over $n-1$ consecutive integers, $-k(n-1)+t$. Now set $v=1$ and $u=-k+1$. Then for $0 \leq t \leq n-1$, $t+(n-1) u$ runs over a set of $n$ consecutive integers, which in combination with the first choice yields a set of $2 n-1$ consecutive integers. As $2 n-1>n-k$, all algebraic shift equivalence classes are realized by a matrix of the form $B$.

(b) Set $u=0, v=n-1$, and $t=1$. We see that $A$ is algebraically shift equivalent to the diagonal matrix by Proposition 1.7, and the resulting matrix $B$ is primitive as $n>2$. 
(c) First, set $v=0$, and $u=n-1$. We thus obtain $n$ consecutive attainable equivalence classes (modulo $2 n-1),\left\{t+(n-1)^{2}\right\}_{0 \leq t \leq n-1}$. As $(n-1)^{2} \equiv n^{2}$ $\bmod (2 n-1)$, the attainable set includes $\left\{t+n^{2}\right\}_{0 \leq t \leq n-1}$.

Now suppose $n=2 d$, that is, $n$ is even. Then $n^{2} \equiv d \bmod 4 d-1$. Thus the integer interval $[d, 3 d-1]$ is attainable. The attainable integers are invariant under multiplication by 2 and -1 modulo $4 d-1$. By considering the smallest integer exceeding 0 that is not attainable, one shows easily that $[1,4 d-2]$ consists of attainable integers.

If $n=2 d+1$, then $n^{2} \equiv-d \bmod 4 d+1$. Set

$$
I=\{a \in \mathbf{Z}|| a \mid \leq n, a \text { is attainable }\} .
$$

Then $I$ is invariant under multiplication by -1 , and contains the integer interval $[-d, d]$. Define $\phi: \mathbf{R} \rightarrow \mathbf{R}$ by $\phi(r)=2 n-1-r$. We note that an integer $j$ is attainable if and only if $\phi(j)$ is. If $3 n / 4 \leq j \leq 2 d+1$, then $-1 \leq \phi(j) \leq d$, so $I$ contains the interval $\left[\left\lceil\frac{3}{4} n\right\rceil, n\right]$. Let $K$ denote the set $K=\{j \in \mathbf{Z} \mid 0 \leq j \leq n, j$ is not attainable $\}$. Then $K \subset\left[d+1, \frac{3}{4} n\right]$, so $\phi(K) \subset[d-1,2 d-1]$. Since $\phi(K) \cap I$ is empty, it follows that $\phi(K) \subseteq K \subseteq$ $\left[d+1, \frac{3}{4} n\right]$; thus $\phi^{l}(K) \subseteq\left[d+1, \frac{3}{4} n\right]$ for any positive integer $l$. However, $\phi\left(\frac{2 n-1}{3}+y\right)=\frac{2 n-1}{3}-2 y$, so $\lim _{l}\left|\phi^{l}(x)-\frac{2 n-1}{3}\right|=\infty$ when $x \neq \frac{2 n-1}{3}$. Hence $K \subseteq\left\{\frac{2 n-1}{3}\right\}$. If $K$ is not empty, $n \equiv 2 \bmod 3$, so $n \equiv 5 \bmod 6$.

Before dealing with $\operatorname{diag}(2 d, 1-2 d)$ and $\left[\begin{array}{cc}6 e+5 & 4 e+3 \\ 0 & -6 e-4\end{array}\right]$ (the really obstinate cases), we dispose of two residual matrices.

Proposition 4.4. (a) All integer matrices whose nonzero eigenvalues are $(2,1)$ (with multiplicity one) are algebraically shift equivalent over $\mathbf{Z}$ to

$$
B=\left[\begin{array}{llll}
1 & 0 & 0 & 1 \\
0 & 1 & 0 & 1 \\
0 & 1 & 1 & 0 \\
1 & 0 & 1 & 0
\end{array}\right]
$$

(b) The matrix $\operatorname{diag}(2,-1)$ is algebraically shift equivalent over $\mathbf{Z}$ to

$$
B=\left[\begin{array}{llll}
0 & 1 & 1 & 0 \\
0 & 1 & 0 & 1 \\
1 & 0 & 0 & 1 \\
1 & 0 & 1 & 0
\end{array}\right]
$$

Proof. (a) Over $\mathbf{Z}$, every matrix is algebraically shift equivalent to a nonsingular matrix; by Proposition 1.7, when the eigenvalues are 2 and 1, the matrix is algebraically shift equivalent (even conjugate) to the diagonal matrix. So it suffices to check that $B$ has $\{2,1,0,0\}$ as its spectrum.

(b) We have a sequence of algebraic shift equivalences:

$$
B \rightarrow\left[\begin{array}{cccc}
0 & 1 & 1 & 0 \\
0 & 1 & 1 & 1 \\
1 & 0 & 1 & 1 \\
0 & 0 & 0 & -1
\end{array}\right] \rightarrow\left[\begin{array}{cccc}
1 & 1 & 1 & 0 \\
0 & 0 & 0 & 1 \\
1 & 0 & 1 & 1 \\
0 & 0 & 0 & -1
\end{array}\right] \rightarrow\left[\begin{array}{ccc}
1 & 1 & -1 \\
1 & 1 & 1 \\
0 & 0 & -1
\end{array}\right] \rightarrow\left[\begin{array}{cc}
2 & 0 \\
0 & -1
\end{array}\right]
$$

Add the fourth column of $B$ to its third, and subtract the third row from the fourth; this yields the second matrix. Add its second column to its first, and subtract the first row from the second; the third matrix results. Add the 
fourth row to the second and subtract the second column from the fourth; then delete the second row and column, and out comes the size 3 matrix. Finally, amalgamate columns one and two.

The following gruesome computation finishes the problem.

Lemma 4.5. The matrices $\operatorname{diag}(2 d, 1-2 d) \quad(d \geq 1)$, and $\left[\begin{array}{cc}6 e+5 & 4 e+3 \\ 0 & -6 e-4\end{array}\right] \quad(e \geq 0)$ are algebraically shift equivalent over $\mathbf{Z}$ to primitive matrices of size 4 .

Proof. If $d$ is even, write it as $d=2 k$; otherwise $d=2 k+1$. The left column in Illustration 1 deals with the case that $d$ is even and describes a series of algebraic strong shift equivalences from a size 4 primitive matrix to a matrix which we shall show is algebraically shift equivalent to the diagonal matrix. The right column implements a similar process when $d$ is odd.

ILlUSTRATION 1. Algebraic shift equivalences in Lemma 4.5.

$$
\begin{gathered}
{\left[\begin{array}{cccc}
1 & k & 3 k-1 & 0 \\
1 & 0 & 1 & 4 k-2 \\
1 & 0 & 0 & 4 k-1 \\
1 & 0 & 4 k-1 & 0
\end{array}\right]} \\
{\left[\begin{array}{cccc}
1 & 4 k-1 & 3 k-1 & 0 \\
1 & 4 k-1 & 1 & 4 k-2 \\
0 & 0 & -1 & 1 \\
0 & 0 & 4 k-2 & 2-4 k
\end{array}\right]} \\
{\left[\begin{array}{cccccc}
4 k & 7 k-2 & (4 k-2)(4 k-1) \\
0 & -1 & 1 & 1 \\
0 & 4 k-2 & 2-4 k
\end{array}\right]} \\
{\left[\begin{array}{ccccc}
4 k & 7 k-2-(4 k-2)^{2}(4 k-1) \\
0 & 1-4 k
\end{array}\right]}
\end{gathered}
$$

To get from the first to the second matrices, subtract the second row from the third and fourth, and add the third and fourth columns to the second. Do the obvious row and column operations to eliminate the second row and column, to obtain the third matrices. From the third matrices, eliminate the third row and column again with the obvious operation; this yields the fourth matrices.

One checks that the $(1,2)$ entry of the size 2 matrices are divisible by $2 n-$ $1=4 d-1$, which will conclude the proof for the case $\operatorname{diag}(2 d, 1-2 d)$.

Now write $t=6 e+4=2 f$ and let $a$ be an integer such that $0<a \leq t$; we will choose a suitable value for $a$. Consider the sequence of algebraic shift 
equivalences,

$$
\begin{aligned}
{\left[\begin{array}{cccc}
1 & a & t-a & 0 \\
1 & 0 & 1 & t-1 \\
1 & 0 & 0 & t \\
1 & 0 & t & 0
\end{array}\right] \rightarrow\left[\begin{array}{cccc}
1 & a & t-a & 0 \\
1 & 0 & t & t-1 \\
1 & 0 & t & t \\
0 & 0 & 0 & -t
\end{array}\right] } \\
\rightarrow\left[\begin{array}{cccc}
1 & t & t-a & 0 \\
1 & t & t & t-1 \\
0 & 0 & 0 & 1 \\
0 & 0 & 0 & -t
\end{array}\right] \rightarrow\left[\begin{array}{ccc}
1 & t & t-a \\
1 & t & t-t(t-1) \\
0 & 0 & -t
\end{array}\right] \\
\rightarrow\left[\begin{array}{ccc}
t+1 & -t^{3}+2 t^{2}+t-a \\
0 & -t
\end{array}\right] .
\end{aligned}
$$

Note that with our choice of values for $a$, the left matrix is primitive. Setting $t=2 f$, then modulo $2 n-1=2 t+1$, we have $-t^{3}+2 t^{2}+t-a \equiv 2 f^{2}-a$. If $f$ is even, $2 f^{2} \equiv-f / 2=-(n-1) / 4$; set $a=(2 n-3) / 3-(n-1) / 4$. On the other hand, if $f=2 g+1$, then $2 n-1=8 g+5$ and $2 f^{2}-a \equiv-5 g-3-a$ $\bmod 8 g+5$; set $a=\frac{2}{3}(2 n-1)-5 g-3$; then $0<a=(g+1) / 3<t$.

In most of the cases, the constructions of this section yield primitive matrices of minimum size in an algebraic shift equivalence class. In particular, for the case of two positive eigenvalues, the constructions of 4.2-4.4 are optimal with respect to size (see Corollary 5.1) and specifically, size 4 in Theorem 4.1 is least possible.

\section{LOWER BOUNDS ON MATRIX SIZES}

What is the smallest size for a primitive matrix to realize a given algebraic shift equivalence class (over the ring $S=\mathbf{Z}$ )? In this section, we establish lower bounds in special cases. Williams [W2] gave an example of a singular primitive integer matrix not algebraically shift equivalent to a nonsingular primitive matrix (also see [El], where a similar example with a stronger property is exhibited, with a different end in view).

Our best results emanate from the conjugacy invariant discussed in [H3], and mentioned en passant in [H1, p. 61]. Let $A$ be an integral matrix of size $n$ whose spectral radius $\lambda$ is an integer and a simple root of the characteristic polynomial of $A$. Let $u$ and $v$ be left and right unimodular eigenvectors of $A$ for $\lambda$ (a vector is unimodular if it consists of integers whose greatest common divisor is 1$)$. The function defined by $\eta(A)=|u \cdot v|$ is integer-valued and an invariant with respect to conjugacy by elements of $\operatorname{GL}(n, \mathbf{Z})$ (observe that $u$ and $v$ are unique up to $\operatorname{sign}$ ). If $A$ is $\operatorname{GL}(n, \mathbf{Z})$-conjugate to a primitive matrix, then the Perron-Frobenius theorem forces $\eta(A) \geq n$.

Corollary 5.1. A diagonal integral matrix of rank 2 or more is not $\mathrm{GL}(\cdot, \mathbf{Z})$ conjugate to a primitive matrix.

Proof. The value of $\eta$ at a diagonal matrix is obviously 1 .

The next, sharper constraint derives from the construction in $[\mathrm{H} 3$, Theorem 1.4].

Proposition 5.2. Suppose that $A$ is an integral matrix whose spectral radius $\lambda$ is an integer and a simple root of the characteristic polynomial $\chi_{A}$. Let $k$ denote 
the multiplicity of 0 as a root of $\chi_{A}$, and suppose the size of $A$ is $n+k$. Then $A$ is algebraically shift equivalent to a size $n$ matrix $B$ such that $\eta(A) \leq \lambda^{k} \eta(B)$. Proof. To begin, suppose that $k=1$. Then $A$ is $\mathrm{GL}(n+1, \mathbf{Z})$-conjugate to a matrix $\left[\begin{array}{ll}B & x \\ 0 & 0\end{array}\right]$ where $B$ has size $n, B$ is algebraically shift equivalent to $A$, and the first column of $B$ is the transpose of $\left(\begin{array}{lllll}\lambda & 0 & 0 & \cdots & 0\end{array}\right)$. If $u B=\lambda u$ with $u_{1}$ as its first entry, then $\left(\lambda u u_{1} x\right) A=\lambda\left(\lambda u u_{1} x\right)$. The right eigenvectors of $A$ and $B$ are the first standard basis elements. Hence $\eta(A) \leq \lambda \eta(B)$. The general case follows by induction on $k$.

Corollary 5.3. Any primitive integer matrix algebraically shift equivalent to either $\operatorname{diag}(2,1)$ or $\operatorname{diag}(2,-1)$ must be of size 4 or more.

Proof. Let $A$ be the primitive matrix of size $2+k$ and let $B$ be the size 2 matrix obtained from Proposition 5.2. It follows from Proposition 1.2 and Proposition 1.7 that as $B$ is algebraically shift equivalent to one of $\operatorname{diag}(2, \pm 1)$ and is of the same size, it must be $\mathrm{GL}(2, \mathbf{Z})$-equivalent to it. Hence $\eta(B)=1$. By Proposition 5.2,

$$
2+k \leq \eta(A) \leq 2^{k} \eta(B)=2^{k},
$$

so $k \geq 2$.

Proposition 5.4. Let $\lambda$ be a positive integer and let $B$ be an element of $\mathrm{GL}(k, \mathbf{Z})$ whose spectral radius is strictly less than $\lambda$. If the matrix $A=\lambda \oplus B$ is algebraically shift equivalent (over $\mathbf{Z}$ ) to a primitive matrix of size $k+1+m$, then $\lambda^{m} \geq k+1+m$.

Proof. Suppose that $C$ is a matrix of size $k+1$ algebraically shift equivalent to $A$. Then $C$ is conjugate via $\operatorname{GL}(k+1, \mathbf{Z})$ to a matrix $D=\left[\begin{array}{ll}\lambda & 0 \\ v & B\end{array}\right]$. As $\lambda$ is not in the spectrum of $B$, an algebraic shift equivalence between $A$ and $D$ involves an equation

$$
\left[\begin{array}{ll}
\lambda & 0 \\
0 & B
\end{array}\right]\left[\begin{array}{ll}
a & 0 \\
x & U
\end{array}\right]=\left[\begin{array}{ll}
a & 0 \\
x & U
\end{array}\right]\left[\begin{array}{ll}
\lambda & 0 \\
v & B
\end{array}\right]
$$

where $U$ belongs to $\operatorname{GL}(k, \mathbf{Z})$ and commutes with $B$. Then $B x=\lambda x+U v$, so $v=(B-\lambda \mathbf{I}) U^{-1} x$. By Proposition 1.1, $D$ is conjugate to $A$. Thus $\eta(C)=$ $\eta(A)=1$, and Proposition 5.2 applies.

Example 5.5. Suppose that $B$ in Proposition 5.4 is a cyclic permutation matrix $P$. Fix $\lambda$; then $m$ (the multiplicity of zero as an eigenvalue of the primitive matrix) goes to infinity with $k$. On the other hand, if $\lambda \geq 3$, the matrix

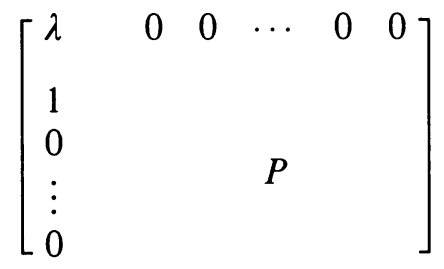

is $\mathrm{GL}(n+1, \mathbf{Z})$-conjugate to a nonsingular primitive matrix (add the first column to each of the others, and subtract the other rows from the first).

Example 5.5 illustrates the constraints that the algebraic shift equivalence class places on the size of an integral primitive matrix, for fixed nonzero spec- 
trum. We now consider primitive matrices algebraically shift equivalent (over $\mathbf{Z})$ to nonsingular matrices of size 2 .

Lemma 5.6. Let $A$ be a singular primitive matrix of size 3 over $\mathbf{Z}$ that has trace 1. If any off-diagonal entry is zero, then $A$ is strongly shift equivalent to $\left[\begin{array}{ll}1 & M \\ 1 & 0\end{array}\right]$ for an integer $M$.

Proof. Write $A=\left(a_{i j}\right)$, where $a_{11}=1$ and $a_{22}=a_{33}=0$. If $a_{21}=a_{23}=0$, delete the second row and column, and we are done. We may assume that the greatest common divisor of $a_{31}$ and $a_{32}$ is 1 (if not, divide row three and multiply column three by the greatest common divisor-this is a strong shift equivalence of $\operatorname{lag} 1)$.

If $a_{21}=0 \neq a_{23}$, computing the determinant, we deduce $a_{23}\left(a_{32}-a_{31} a_{12}\right)=$ 0 , so $a_{32}=a_{31} a_{12}$. The greatest common divisor condition forces $a_{31}=1$. Now subtract $a_{12}$ copies of the first column from the second, and add the same number of copies of the second row to the first; then delete the second row and column. This yields the matrix $\left[\begin{array}{cc}1 & a_{13}+a_{12} a_{23} \\ 1 & 0\end{array}\right]$.

If $a_{21}$ vanishes, we apply the same process to the transpose of $A$; we note that $\left[\begin{array}{ll}1 & M \\ 1 & 0\end{array}\right]$ is strongly shift equivalent to its transpose. If $a_{31}$ or $a_{13}$ vanish, we may reduce to the previous case by conjugating $A$ with a permutation matrix.

Finally, we may assume that all of $a_{12}, a_{21}, a_{13}, a_{31}$ are not zero but $a_{32}=0$. The determinant being zero, $a_{23}=0$ as well. Divide the second and third rows by $a_{21}, a_{13}$ respectively, and multiply the second and third rows correspondingly. Then the second and third rows are equal, and we amalgamate to obtain a matrix of the desired form.

The matrix $\left[\begin{array}{ll}1 & M \\ 1 & 0\end{array}\right]$ is a companion matrix; hence if it has integer eigenvalues, it is conjugate to $\left[\begin{array}{cc}n & 1 \\ 0 & 1-n\end{array}\right]$. In particular, it cannot be algebraically shift equivalent to a diagonal matrix (as the eigenvalues are relatively prime).

Let $B$ be a trace 1 matrix of size 2 that is not algebraically shift equivalent to its companion matrix but is algebraically shift equivalent to a primitive matrix $A$ of size 3. By Lemma 5.6, $A$ has only two zero entries (on the diagonal). In particular, detailed computations can be used to show that each of the matrices

$$
\left[\begin{array}{cc}
2 & 3 \\
3 & -1
\end{array}\right] \quad\left[\begin{array}{cc}
5 & 3 \\
0 & -4
\end{array}\right] \quad\left[\begin{array}{cc}
3 & 5 \\
7 & -2
\end{array}\right]
$$

is not algebraically shift equivalent to a primitive matrix of size 2 or 3 . Note that the middle matrix is one of those dealt with in Lemma $4.5(e=0)$.

One of the difficulties in using Theorem 3.1 is that we do not know enough about starting the process. For example, over $S=\mathbf{Z}$, we have no results that determine whether a matrix with irreducible characteristic polynomial is algebraically shift equivalent to a primitive matrix. In particular, suppose that $A$ is size 2 with irrational eigenvalues and positive trace. If $A$ has positive determinant, then $A$ is $\mathrm{GL}(2, \mathrm{Z})$ y-equivalent to a primitive matrix [BH]. If the determinant of $A$ is negative, we do not know if $A$ is algebraically shift equivalent to a primitive matrix at all (even though the companion matrix is automatically primitive). Specifically, we were not able to realize $\left[\begin{array}{cc}2 & 3 \\ 3 & -1\end{array}\right]$ above as a primitive matrix. 


\section{REFERENCES}

[Al] G. Almkvist, Endomorphisms of finitely generated projective modules over a commutative ring, Ark. Mat. 11 (1973), 263-301.

[As] J. Ashley, Resolving factor maps for shifts of finite type with equal entropy, Ergodic Theory Dynamical Systems 11 (1991), 219-240.

[Ba] H. Bass, Algebraic K-theory, Benjamin, New York, 1973.

[B1] K. A. Baker, Strong shift equivalence of 2 by 2 matrices of nonnegative integers, Ergodic Theory Dynamical Systems 3 (1983), 501-508.

[B2] _ Strong shift equivalence and shear adjacency of nonnegative square integer matrices, Linear Algebra Appl. 93 (1987), 131-147.

[BMT] M. Boyle, B. Marcus, and P. Trow, Resolving maps and the dimension group for shifts of finite type, Mem. Amer. Math. Soc. No. 377 (1987).

[B1] M. Boyle, Shift equivalence and the Jordan form away from zero, Ergodic Theory Dynamical Systems 4 (1984), 367-379.

[BH] M. Boyle and D. Handelman, The spectra of nonnegative matrices via symbolic dynamics, Ann. of Math. (2) 133 (1991), 249-316.

[BLR] M. Boyle, D. Lind, and D. Rudolph, The automorphism group of a shift of finite type, Trans. Amer. Math. Soc. 306 (1988), 71-114.

[Ef] E. G. Effros, On Williams' problem for positive matrices, unpublished manuscript, 1981.

[El] G. Elliott, On totally ordered groups and $K_{0}$, Proc. Conf. Ring Theory (Waterloo 1978), Lecture Notes in Math., vol. 734, Springer, 1979, pp. 1-50.

[Fi] U. Fiebig, Gyration numbers for involutions of subshifts of finite type. I, II, Forum Math. 4 (1992), 77-108, 183-211.

[H1] D. E. Handelman, Positive matrices and dimension groups affiliated to $C^{*}$-algebras and topological Markov chains, J. Operator Theory 6 (1981), 55-74.

[H2] — Reducible toplogical Markov chains via $K_{0}$-theory and Ext, Contemp. Math., vol. 10 , Amer. Math. Soc., Providence, R.I., 1982, pp. 41-76.

[H3] _ Eventually positive matrices with rational eigenvectors, Ergodic Theory Dynamical Systems 7 (1987), 193-196.

[H4] Strongly indecomposable abelian groups and totally ordered topological Markov chains, unpublished manuscript, 1981.

[KR1] K. H. Kim and F. W. Roush, Some results on the decidability of shift equivalence, J. Combin. Inform. System Sci. 4 (1979), 123-146.

[KR2] _ On strong shift equivalence over a Boolean semiring, Ergodic Theory Dynamical Systems 6 (1986), 81-97.

[KR3] - Williams' conjecture is false for reducible subshifts, J. Amer. Math. Soc. 5 (1992), 213-215.

[KR4] _ Strong shift equivalence of Boolean and rational matrices, Linear Algebra \& Appl. 161 (1992), 153-164.

[KMT] B. Kitchens, B. Marcus, and P. Trow, Eventual factor maps and compositions of closing maps, Ergodic Theory Dynamical Systems 11 (1991), 85-113.

[Kr1] W. Krieger, On dimension functions and topological Markov chains, Invent. Math. 56 (1980), 239-250.

[Kr2] _ On the subsystems of topological Markov chains, Ergodic Theory Dynamical Systems 2 (1982), 195-202.

[MT] B. Marcus and S. Tuncel, The weight-per-symbol polytope and scaffolds of invariants associated with Markov chains, Ergodic Theory Dynamical Systems 11 (1991), 129-180.

[M] J. Milnor, Introduction to algebraic K-theory, Ann. Math. Stud., Princeton Univ. Press, Princeton, NJ, 1971.

[N] M. Newman, Integral matrices, Academic Press, 1978. 
[PT] W. Parry and S. Tuncel, On the stochastic and topological structure of Markov chains, Bull. London Math. Soc. 14 (1982), 16-27.

[PW] W. Parry and R. F. Williams, Block coding and a zeta function for Markov chains, Proc. London Math. Soc. 35 (1977), 483-495.

[Sa] P. Samuel, Théorie algébrique des nombres, Hermann, Paris, 1967.

[W1] R. F. Williams, Classification of one-dimensional attractors, Proc. Sympos. Pure Math., vol. 14, Amer. Math Soc., Providence, R.I., 1970, pp. 341-361.

[W2] _ Classification of subshifts of finite type, Ann. of Math. (2) 98 (1973), 120-153; Errata, Ann. of Math. (2) 99 (1974), 380-381.

[W3] _ Strong shift equivalence of matrices in $\mathrm{GL}(2, \mathbf{Z})$, unpublished manuscript, 1981.

Department of Mathematics, University of Maryland, College Park, Maryland 20742

E-mail address: mmb@math.umd.edu

Department of Mathematics, University of Ottawa, Ottawa, Ontario, Canada Kin 6N5

E-mail address: acadvm1.uottawa.ca 